\title{
Bioinspired Synthesis of the Furopyrazine Alkaloid Hyrtioseragamine A
}

Shi-Wei Kim, Paul A. Hume and Jonathan Sperry*

j.sperry@auckland.ac.nz

SUPPORTING INFORMATION 
Table S1 ${ }^{1} \mathrm{H}$ and ${ }^{13} \mathrm{C}$ NMR spectroscopic comparison of natural $\mathbf{1}$ with synthetic $\mathbf{1}$

DFT Calculations - Computational Methods

(14) ${ }^{1} \mathrm{H}$ NMR (400 MHz, $\left.\mathrm{CDCl}_{3}\right)$

(14) ${ }^{13} \mathrm{C}$ NMR $\left(101 \mathrm{MHz}, \mathrm{CDCl}_{3}\right)$

(S1) ${ }^{1} \mathrm{H}$ NMR (400 MHz, $\left.\left(\mathrm{CD}_{3}\right)_{2} \mathrm{SO}\right)$

(S1) ${ }^{13} \mathrm{C}$ NMR $\left(101 \mathrm{MHz},\left(\mathrm{CD}_{3}\right)_{2} \mathrm{SO}\right)$

S10

(15) ${ }^{1} \mathrm{H}$ NMR (400 MHz, (CD 3$\left.)_{2} \mathrm{SO}\right)$

S11

(15) ${ }^{13} \mathrm{C}$ NMR $\left(101 \mathrm{MHz},\left(\mathrm{CD}_{3}\right)_{2} \mathrm{SO}\right)$

S12

(16) ${ }^{1} \mathrm{H}$ NMR $\left(400 \mathrm{MHz},\left(\mathrm{CD}_{3}\right)_{2} \mathrm{SO}\right)$

S13

(16) ${ }^{13} \mathrm{C}$ NMR (101 MHz, $\left.\left(\mathrm{CD}_{3}\right)_{2} \mathrm{SO}\right)$

S14

(19) ${ }^{1} \mathrm{H}$ NMR (400 MHz, $\left.\mathrm{CDCl}_{3}\right)$

S15

(19) ${ }^{13} \mathrm{C} \mathrm{NMR}\left(101 \mathrm{MHz}, \mathrm{CDCl}_{3}\right)$

S16

(21) ${ }^{1} \mathrm{H}$ NMR $\left(400 \mathrm{MHz},\left(\mathrm{CD}_{3}\right)_{2} \mathrm{SO}\right)$

S17

(21) ${ }^{13} \mathrm{C}$ NMR $\left(101 \mathrm{MHz},\left(\mathrm{CD}_{3}\right)_{2} \mathrm{SO}\right)$

S18

(24) ${ }^{1} \mathrm{H}$ NMR $\left(400 \mathrm{MHz}, \mathrm{CDCl}_{3}\right)$

S19

(24) ${ }^{13} \mathrm{C}$ NMR $\left(101 \mathrm{MHz}, \mathrm{CDCl}_{3}\right)$

S20

(S2) ${ }^{1} \mathrm{H}$ NMR $\left(400 \mathrm{MHz}, \mathrm{CDCl}_{3}\right)$

S21

(S2) ${ }^{13} \mathrm{C} \mathrm{NMR}\left(101 \mathrm{MHz}, \mathrm{CDCl}_{3}\right)$

S22

(25) ${ }^{1} \mathrm{H}$ NMR (400 MHz, $\mathrm{CDCl}_{3}$ )

S23

(25) ${ }^{13} \mathrm{C} \mathrm{NMR}\left(101 \mathrm{MHz}, \mathrm{CDCl}_{3}\right)$

S24

(26) ${ }^{1} \mathrm{H}$ NMR (400 MHz, $\left.\left(\mathrm{CD}_{3}\right)_{2} \mathrm{SO}\right)$

S25

(26) ${ }^{13} \mathrm{C}$ NMR $\left(101 \mathrm{MHz},\left(\mathrm{CD}_{3}\right)_{2} \mathrm{SO}\right)$

S26

(28) ${ }^{1} \mathrm{H}$ NMR (400 MHz, $\left.\left(\mathrm{CD}_{3}\right)_{2} \mathrm{SO}\right)$

S27

(28) ${ }^{13} \mathrm{C}$ NMR $\left(101 \mathrm{MHz},\left(\mathrm{CD}_{3}\right)_{2} \mathrm{SO}\right)$

S28

(29) ${ }^{1} \mathrm{H}$ NMR (400 MHz, CD 3 OD)

S29

(29) ${ }^{13} \mathrm{C}$ NMR (101 MHz, CD 3 OD)

S30

Hyrtioseragamine A (1) ${ }^{1} \mathrm{H}$ NMR $\left(500 \mathrm{MHz}, \mathrm{CD}_{3} \mathrm{OD}\right)$

S31

Hyrtioseragamine A (1) ${ }^{13} \mathrm{C}$ NMR (126 MHz, CD $\left.{ }_{3} \mathrm{OD}\right)$

S32

References

S33 
Table S1. ${ }^{1} \mathrm{H}$ and ${ }^{13} \mathrm{C}$ NMR comparison of natural $\mathbf{1}$ with synthetic $\mathbf{1}$

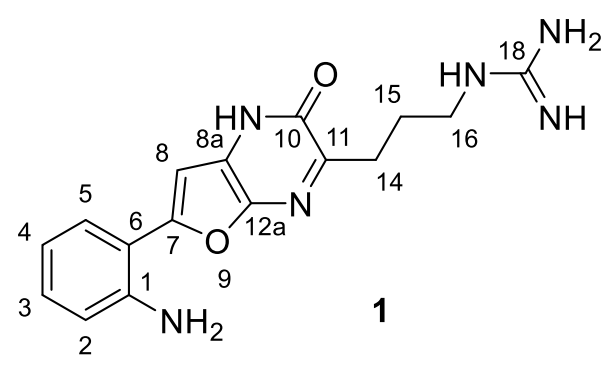

\begin{tabular}{|c|c|c|c|c|c|c|}
\hline position & $\begin{array}{c}\text { Natural } \delta_{C}^{* 1} \\
\left(\mathrm{CD}_{3} \mathrm{OD}, 150 \mathrm{MHz}\right)\end{array}$ & $\begin{array}{c}\text { Synthetic } \delta C^{*} \\
\left(\mathrm{CD}_{3} \mathrm{OD}, 125 \mathrm{MHz}\right)\end{array}$ & $\Delta$ & $\begin{array}{c}\text { Natural } \delta_{\mathrm{H}^{* 1}} \\
\left(\mathrm{CD}_{3} \mathrm{OD}, 600 \mathrm{MHz}\right)\end{array}$ & $\begin{array}{c}\text { Synthetic } \delta_{\mathrm{H}^{*}} \\
\left(\mathrm{CD}_{3} \mathrm{OD}, 500 \mathrm{MHz}\right)\end{array}$ & $\Delta$ \\
\hline 1 & 144.6 & 144.9 & -0.3 & & & \\
\hline 2 & 120.6 & 120.5 & 0.1 & $7.03($ br d, $J=8.0 \mathrm{~Hz})$ & $7.02(\mathrm{dd}, J=8.0,1.3 \mathrm{~Hz})$ & -0.01 \\
\hline 3 & 132.6 & 132.7 & -0.1 & $7.25(\mathrm{ddd}, J=8.0,7.8,1.4 \mathrm{~Hz})$ & $7.28(\mathrm{ddd}, J=8.2,7.3,1.3 \mathrm{~Hz})$ & -0.03 \\
\hline 4 & 121.8 & 121.7 & 0.1 & $6.91(\mathrm{ddd}, J=7.8,7.6,1.0 \mathrm{~Hz})$ & $6.95(\mathrm{~m} /$ unresolved $)$ & -0.04 \\
\hline 5 & 129.9 & 129.9 & 0 & $7.64(\mathrm{dd}, J=7.8,1.4 \mathrm{~Hz})$ & $7.69(\mathrm{dd}, J=7.9,1.6 \mathrm{~Hz})$ & -0.05 \\
\hline 6 & 117.4 & 117.3 & 0.1 & & & \\
\hline 7 & 158.5 & 158.6 & -0.1 & & & \\
\hline 8 & 100.3 & 100.4 & -0.1 & $6.94(\mathrm{~s})$ & $6.99(\mathrm{~s})$ & -0.05 \\
\hline $\mathbf{8 a}$ & 127.8 & 128.0 & -0.2 & & & \\
\hline 10 & 159.1 & 159.1 & 0 & & & \\
\hline 11 & 148.7 & 148.5 & 0.2 & & & \\
\hline $12 \mathbf{a}$ & 148.2 & 148.3 & -0.1 & & & \\
\hline 14 & 31.3 & 31.3 & 0 & $2.91(\mathrm{t}, J=7.2 \mathrm{~Hz})$ & $2.94(\mathrm{t}, J=7.3 \mathrm{~Hz})$ & -0.03 \\
\hline 15 & 27.8 & 27.8 & 0 & 2.08 (quin, $J=7.2 \mathrm{~Hz}$ ) & 2.10 (quin, $J=7.2 \mathrm{~Hz}$ ) & -0.02 \\
\hline 16 & 42.7 & 42.7 & 0 & $3.34(\mathrm{t}, J=7.2 \mathrm{~Hz})$ & under residual $\mathrm{MeOH}$ & \\
\hline 18 & 159.6 & 159.5 & 0.1 & & & \\
\hline
\end{tabular}

*Spectrum referenced to residual methanol $3.35 \mathrm{ppm}\left({ }^{1} \mathrm{H}\right.$ nuclei) and $49.8 \mathrm{ppm}\left({ }^{13} \mathrm{C}\right.$ nuclei $)$ 


\section{DFT Calculations}

\section{Computational Methods}

DFT calculations were performed using the Gaussian 09 software package. ${ }^{2}$ Geometry optimizations of starting materials, transition states and products were performed using the M06- $2 \mathrm{X}^{3}$ exchangecorrelation functional and the $6-31 \mathrm{G}+(\mathrm{d}, \mathrm{p})$ basis set. All calculations employed tight convergence criteria and an ultrafine integration grid with symmetry turned off. Energetic minima were characterised by normal mode vibrational frequency calculations, which revealed zero imaginary frequencies in all cases. All calculations employed a polarizable continuum (PCM) model with water as the solvent. Predicted free energy values were calculated as the sum of the electronic energy and the relevant thermal correction to the energy at $298.15 \mathrm{~K}$.

\section{Cartesian Coordinates $(\AA ̊)$, Enthalpy (H, Ha), and Gibbs Free Energy (G, Ha).}

\section{P1}

$H=-894.014653, G=-894.079858$<smiles>C[C@H]1NC(=O)[C@H](CC(=O)c2ccccc2N)NC1=O</smiles>
C $\quad-3.91232900 \quad-0.11442100 \quad 0.66798700$
C $\quad-5.08631800 \quad-0.18903400 \quad-0.21167300$
C $\quad-4.97388600 \quad-0.76619800 \quad-1.49369400$
C $\quad-6.04393700 \quad-0.84853900-2.35710400$
C $\quad-7.28407800 \quad-0.33772900-1.94344300$
C $\quad-7.43464300 \quad 0.23166300 \quad-0.70005200$
C $\quad-6.34851000 \quad 0.32254100 \quad 0.20229200$
$\begin{array}{llll}\text { O } & -3.95904600 & 0.37998100 & 1.79004700\end{array}$
C $\quad-0.26363300 \quad-1.31676600 \quad 0.79450000$
C $\quad-1.48573300 \quad-0.52173100 \quad 1.22602900$
C $\quad-2.58591900 \quad-0.67605800 \quad 0.17221200$
N $\quad 0.83819100 \quad-0.61985100 \quad 0.46969400$
$\begin{array}{llll}\text { C } & 0.94387400 & 0.82580300 & 0.31115500\end{array}$
C $\quad-0.11643700 \quad 1.57351100 \quad 1.10129500$
N $\quad \begin{array}{llll}-1.18541500 & 0.87142100 & 1.51445800\end{array}$
C $\quad 0.88362100 \quad 1.24769600 \quad-1.15964800$
$\begin{array}{llll}\text { O } & 0.01929300 & 2.77024400 & 1.31672900\end{array}$
O $\quad-0.31941400 \quad-2.53823800 \quad 0.76234400$
$\mathrm{N} \quad-6.55906300 \quad 0.87119500 \quad 1.42516300$

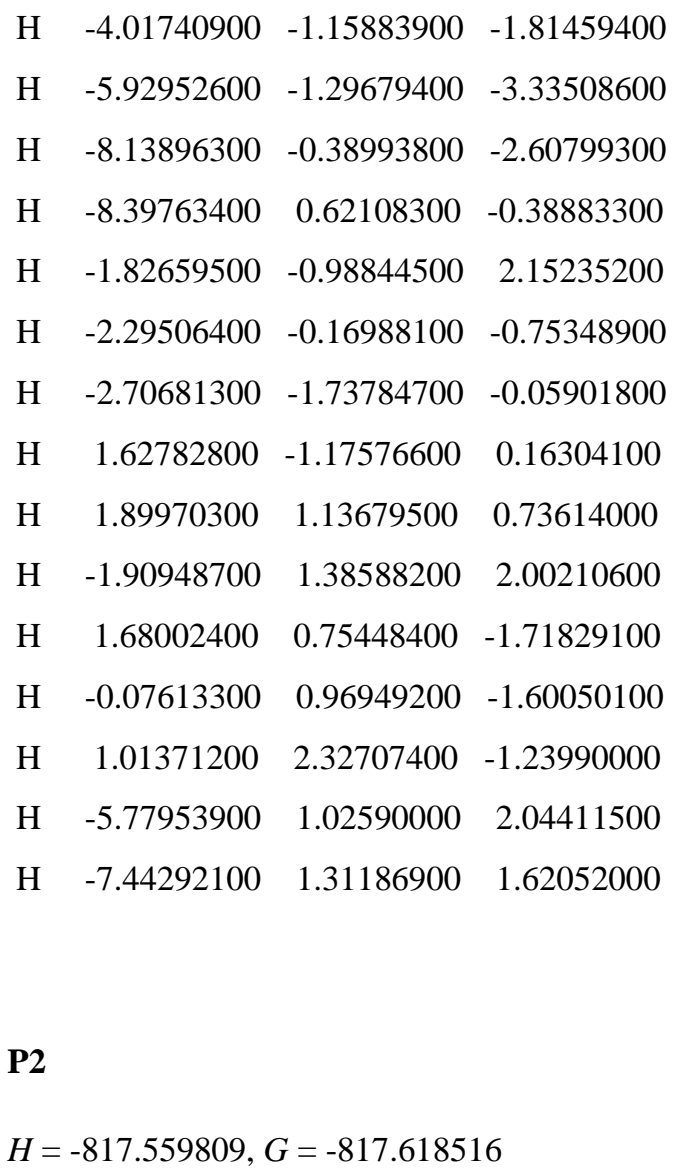




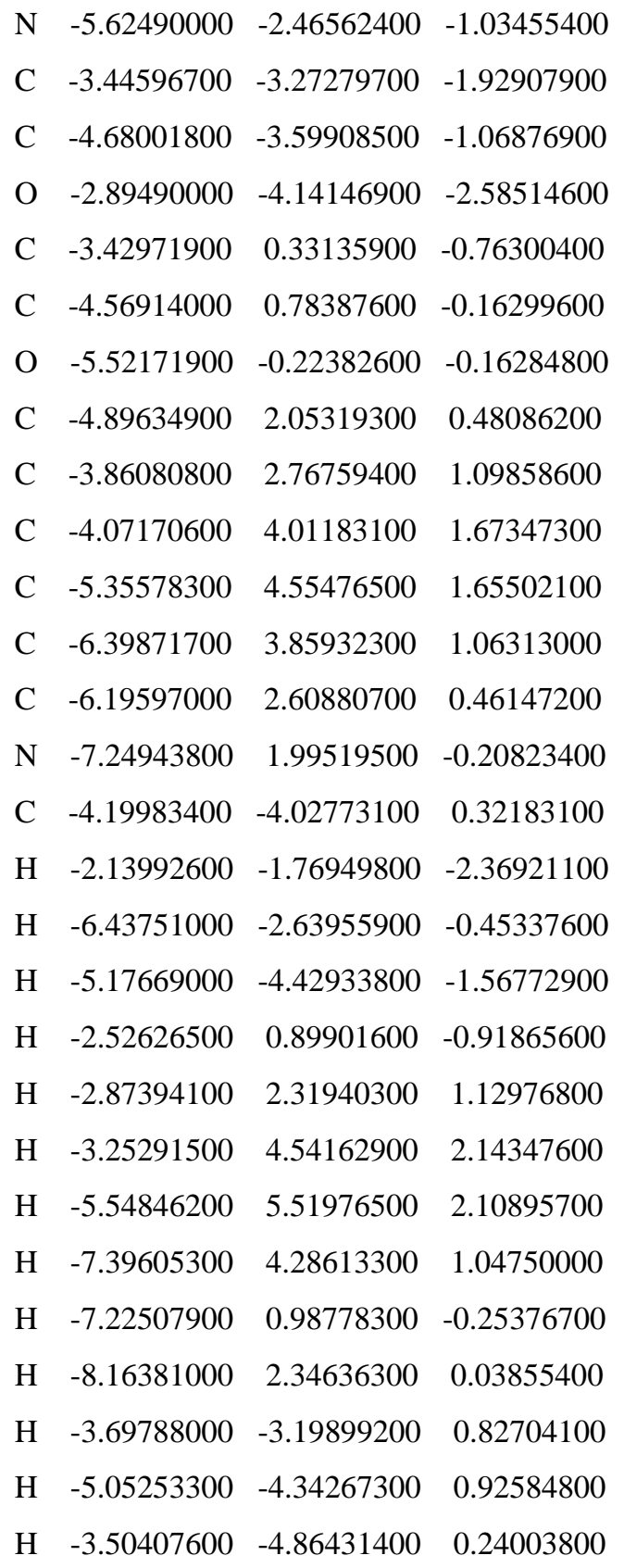

P3

$H=-892.803143, G=-892.867587$<smiles>C[C@H]1NC(=O)/C(=C\C(=O)c2ccccc2N)NC1=O</smiles>
C $\quad-6.30900400 \quad 0.31545400 \quad-0.23294700$
C $\quad-6.30898100 \quad-1.18931000 \quad-0.17308100$

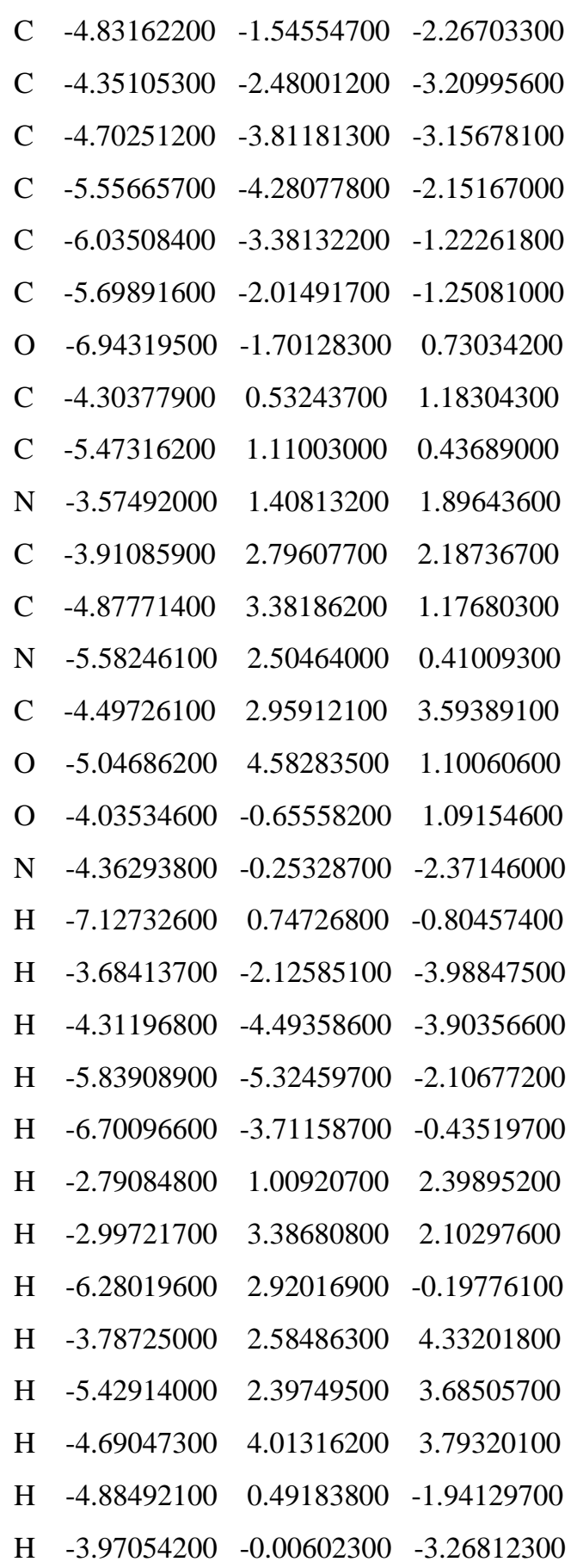

P4

$H=-892.787462, G=-892.847655$<smiles>C[C@H]1N=C2OC(O)(c3ccccc3N)C=C2NC1=O</smiles> 


$$
\begin{array}{lrrr}
\mathrm{C} & -2.81487800 & -1.19792400 & -0.94896600 \\
\mathrm{C} & -3.62168600 & 0.08137800 & -0.87534400 \\
\mathrm{C} & -4.35033800 & 0.08303400 & 1.58488600 \\
\mathrm{C} & -5.38423800 & 0.15892900 & 2.52952400 \\
\mathrm{C} & -6.71280900 & 0.23215000 & 2.14394800 \\
\mathrm{C} & -7.04946000 & 0.21927000 & 0.79385500 \\
\mathrm{C} & -6.03373600 & 0.15849800 & -0.15144100 \\
\mathrm{C} & -4.68651900 & 0.11749700 & 0.21394500 \\
\mathrm{O} & -4.14152600 & 0.31716800 & -2.14452400 \\
\mathrm{C} & -1.39783600 & 0.57305700 & -0.64675500 \\
\mathrm{C} & -1.52570100 & -0.88139300 & -0.85245700 \\
\mathrm{~N} & -0.34525900 & 1.24382100 & -0.49942500 \\
\mathrm{C} & 0.91937800 & 0.51649500 & -0.58380700 \\
\mathrm{C} & 0.87090200 & -1.01020400 & -0.69994000 \\
\mathrm{~N} & -0.35473500 & -1.60473300 & -0.86917400 \\
\mathrm{C} & 1.74004900 & 1.06671700 & -1.75543100 \\
\mathrm{O} & 1.88827400 & -1.67128700 & -0.66494000 \\
\mathrm{O} & -2.63514100 & 1.10953300 & -0.59561000 \\
\mathrm{H} & 1.46981500 & 0.71095000 & 0.34141400 \\
\mathrm{~N} & -3.03295500 & 0.03404100 & 2.03971500 \\
\mathrm{H} & -3.27529100 & -2.16265800 & -1.10008300 \\
\mathrm{H} & -5.12445700 & 0.14803200 & 3.58277300 \\
\mathrm{H} & -7.48585800 & 0.28491900 & 2.90160300 \\
\mathrm{H} & -8.08437000 & 0.25472100 & 0.47873500 \\
\mathrm{H} & -6.29653800 & 0.13419900 & -1.20146000 \\
\mathrm{H} & -4.63350100 & 1.14858700 & -2.13028400 \\
\mathrm{H} & -0.37177300 & -2.61327000 & -0.96989500 \\
\mathrm{H} & 1.22784600 & 0.87018800 & -2.69969300 \\
\mathrm{H} & 1.85227300 & 2.14421500 & -1.63664600 \\
\mathrm{H} & 2.72492000 & 0.60178500 & -1.77755900 \\
\mathrm{H} & -2.40248300 & -0.53011300 & 1.48699200 \\
& -2.95916700 & -0.21460100 & 3.01724400
\end{array}
$$$$
\text { P5 }
$$$$
H=-816.391243, G=-816.448442
$$<smiles>Cc1nc2oc(-c3ccccc3N)cc2[nH]c1=O</smiles>

$$
\begin{array}{lrrr}
\mathrm{C} & -3.43810500 & -0.90487600 & -0.76779000 \\
\mathrm{~N} & -2.70031500 & -1.90679100 & -1.33228500 \\
\mathrm{C} & -4.59033100 & -1.26876200 & -0.12143300 \\
\mathrm{~N} & -5.07458100 & -2.50561800 & 0.01580200 \\
\mathrm{C} & -3.09604000 & -3.22420800 & -1.25254800 \\
\mathrm{C} & -4.36622600 & -3.46189100 & -0.52158400 \\
\mathrm{O} & -2.43530600 & -4.12668600 & -1.75592600 \\
\mathrm{C} & -3.32991800 & 0.50878100 & -0.67850400 \\
\mathrm{C} & -4.43274300 & 0.90751700 & 0.03260400 \\
\mathrm{O} & -5.20752200 & -0.16428000 & 0.37531500 \\
\mathrm{C} & -4.84913100 & 2.23621000 & 0.47076300 \\
\mathrm{C} & -3.84924200 & 3.20006800 & 0.67074000 \\
\mathrm{C} & -4.15262200 & 4.49899500 & 1.03830100 \\
\mathrm{C} & -5.49129800 & 4.85073200 & 1.22313800 \\
\mathrm{C} & -6.49379000 & 3.91519300 & 1.03981300 \\
\mathrm{C} & -6.20407600 & 2.59372900 & 0.65940100 \\
\mathrm{~N} & -7.24942800 & 1.72283400 & 0.40396900 \\
\mathrm{C} & -4.81435800 & -4.88274900 & -0.42558500 \\
\mathrm{H} & -1.83633300 & -1.71142200 & -1.82560600 \\
\mathrm{H} & -2.56656600 & 1.14723500 & -1.09115800 \\
\mathrm{H} & -2.81361900 & 2.90430500 & 0.54728900 \\
\mathrm{H} & -3.36288300 & 5.22277400 & 1.19219100 \\
\mathrm{H} & -5.75391400 & 5.85971700 & 1.51880700 \\
\mathrm{H} & -7.53178600 & 4.19530300 & 1.18324700 \\
\mathrm{H} & -7.06859300 & 0.73736000 & 0.51507900 \\
\mathrm{H} & -8.14364600 & 2.00733400 & 0.77626500 \\
\mathrm{H} & -4.05401100 & -5.48838600 & 0.07367200 \\
\mathrm{H} & -5.74928200 & -4.93682600 & 0.12824600 \\
\mathrm{H} & -4.95163500 & -5.30649100 & -1.42352300
\end{array}
$$

$$
\begin{aligned}
& \mathbf{H}_{2} \mathbf{O} \\
& H=-76.403638, G=-76.425715 \\
& \begin{array}{llll}
\mathrm{O} & -0.33962100 & -1.05727200 & 0.00000000
\end{array} \\
& \mathrm{H} \quad 0.62063900 \quad-1.01540600 \quad 0.00000000 \\
& \mathrm{H} \quad-0.62070100 \quad-0.13811300 \quad 0.00000000
\end{aligned}
$$




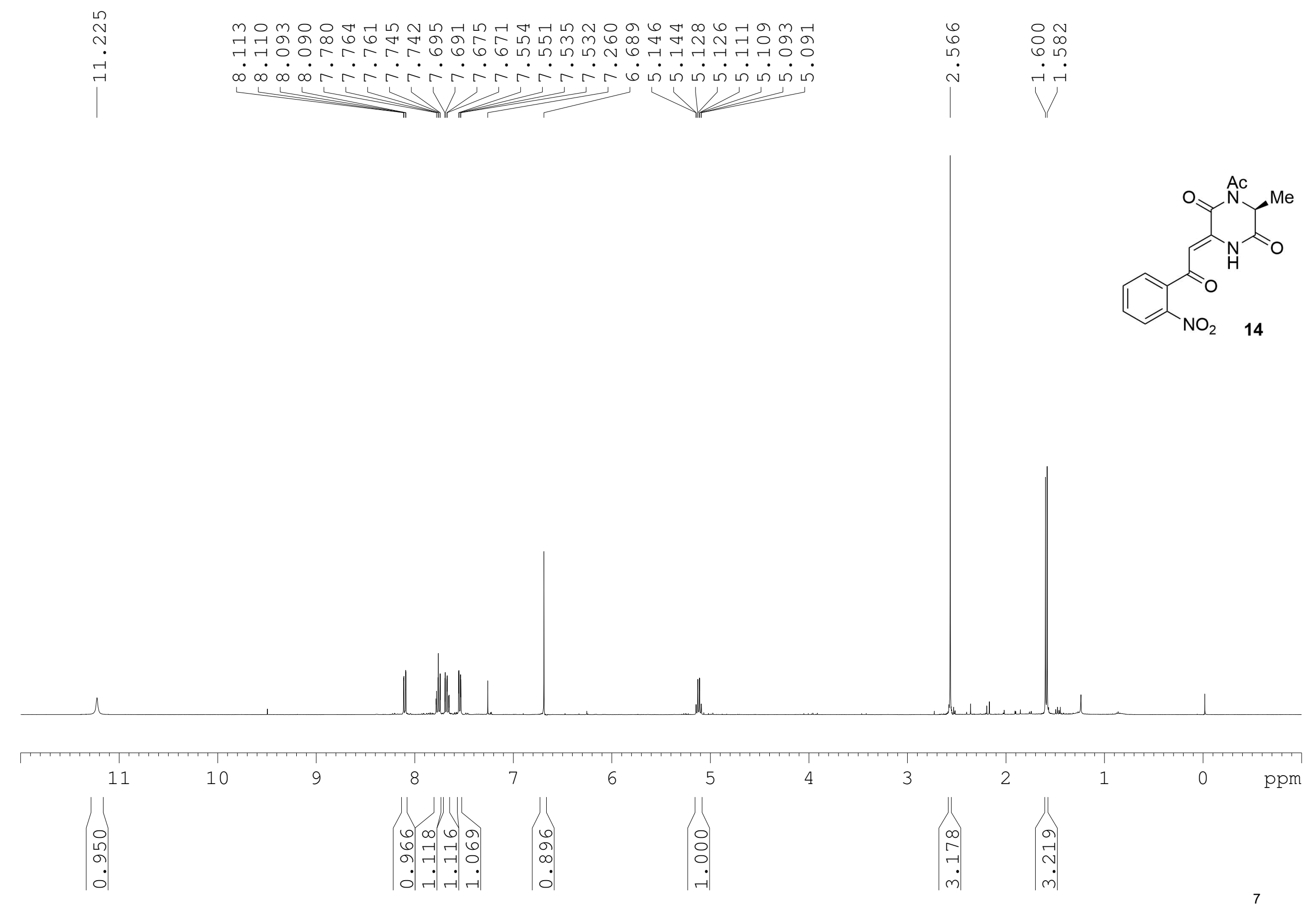




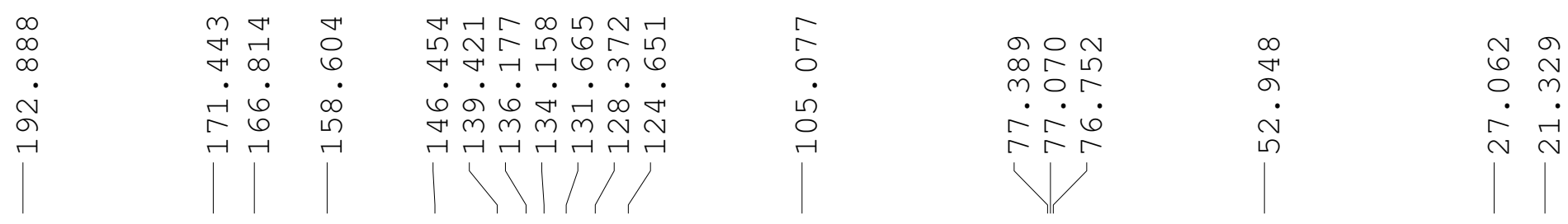

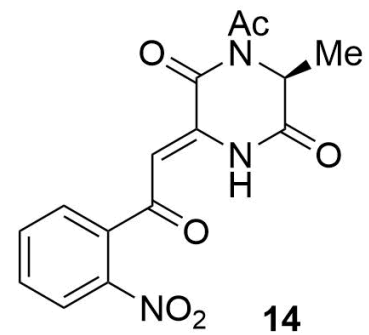

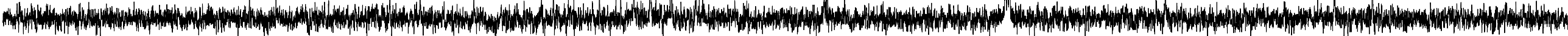

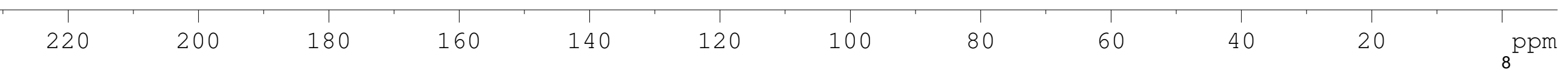




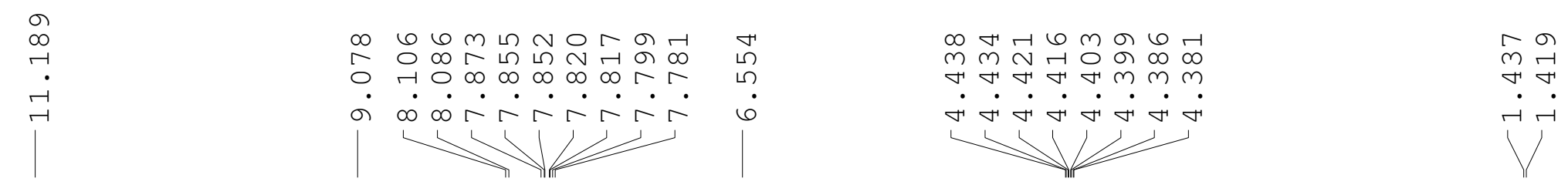

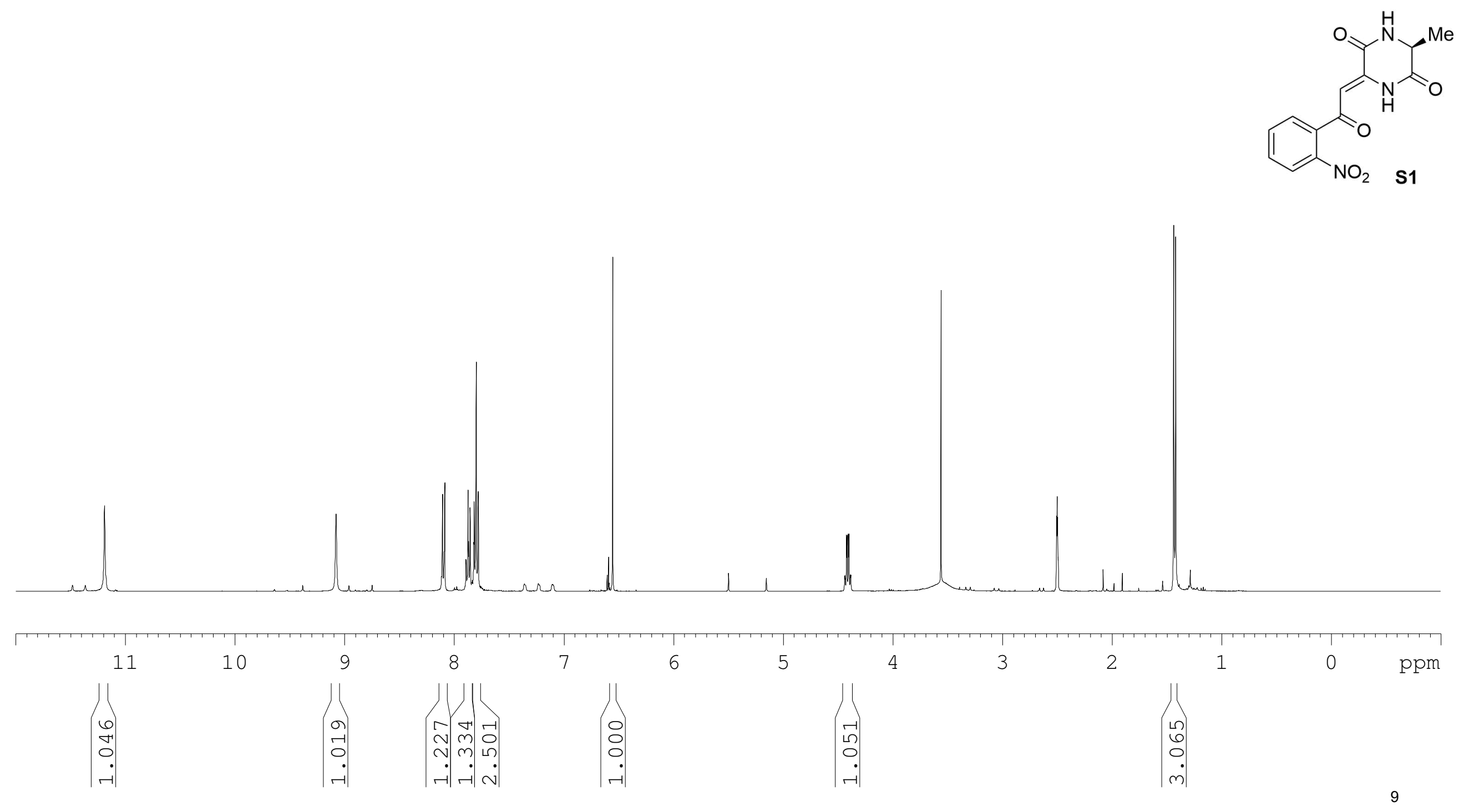



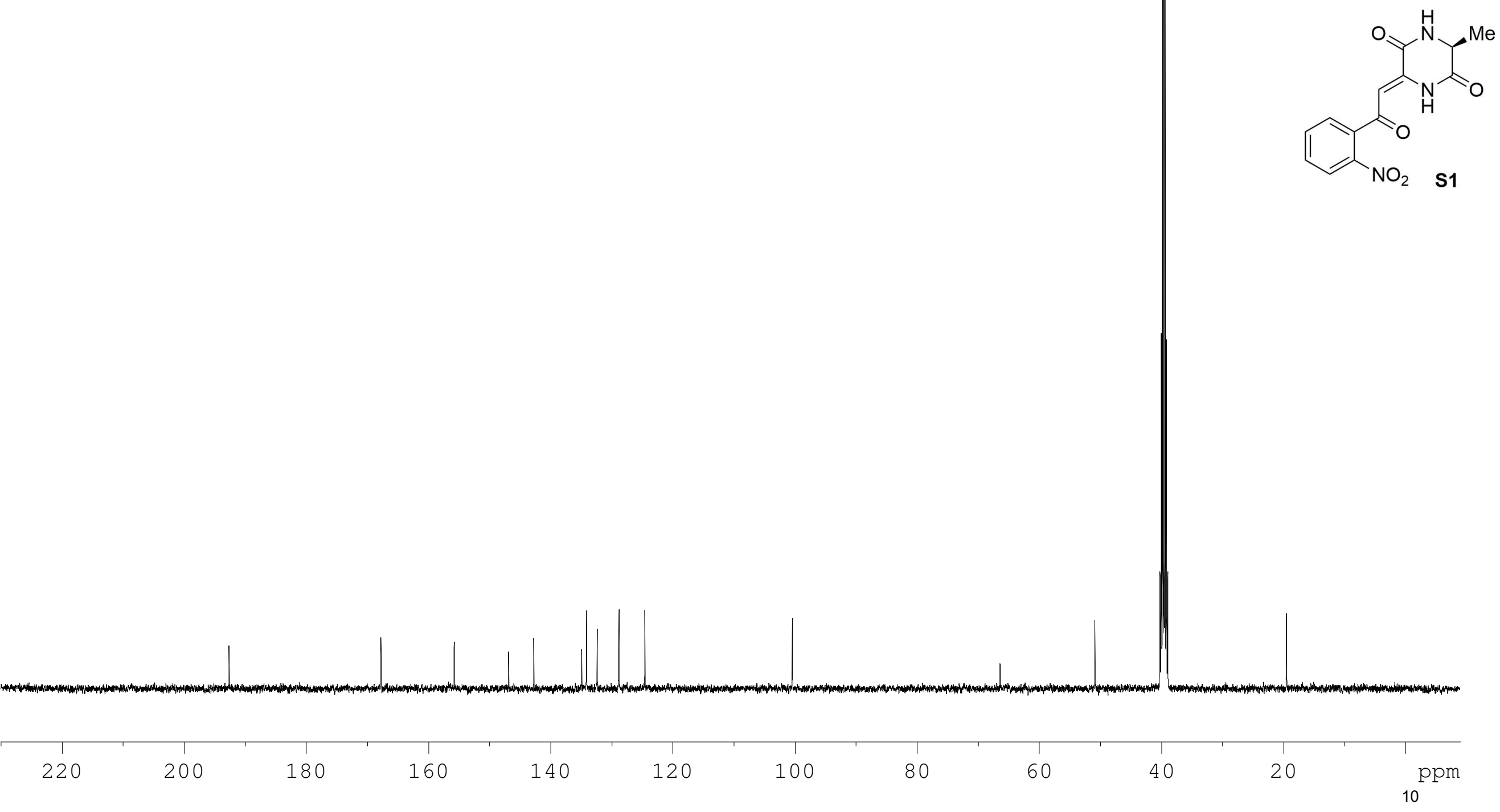


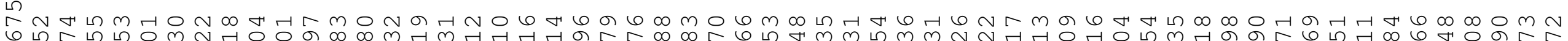

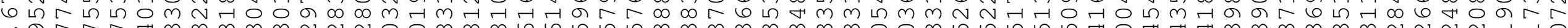

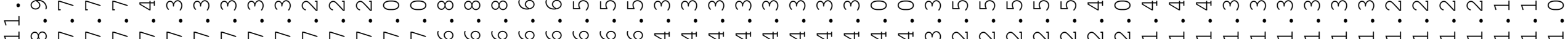
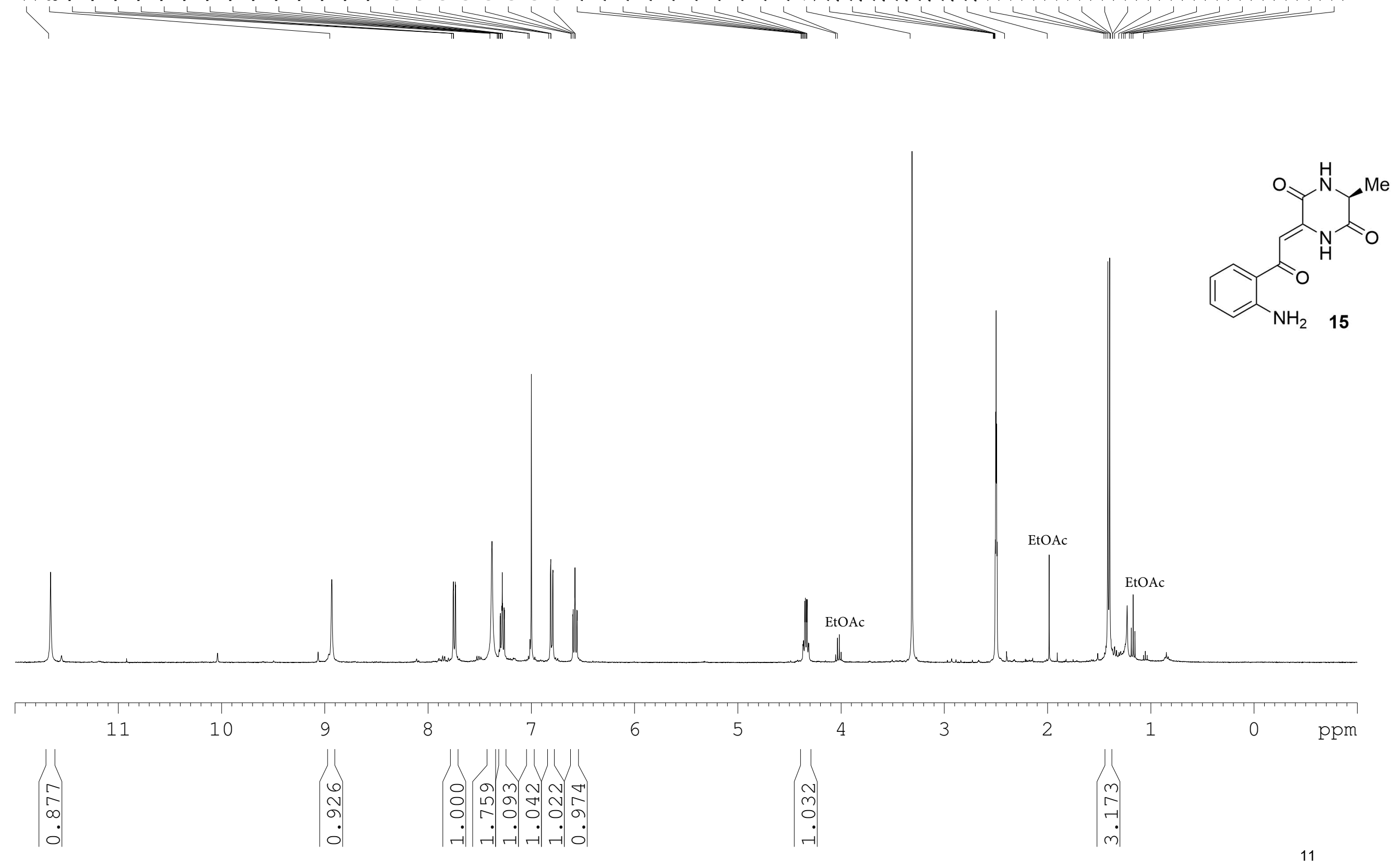


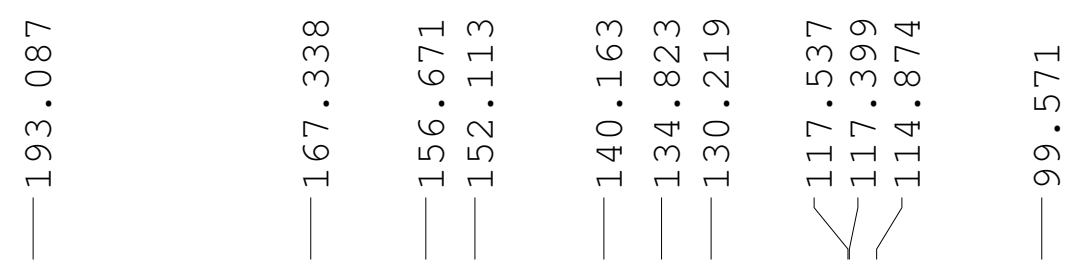

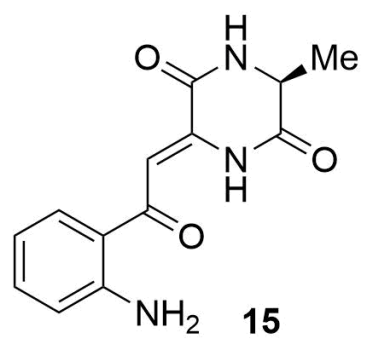

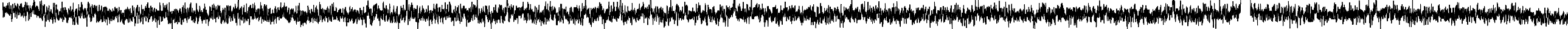

\begin{tabular}{|c|c|c|c|c|c|c|c|c|c|c|c|}
\hline 1 & $T$ & $T$ & 1 & $T$ & 1 & $T$ & $\uparrow$ & $T$ & $T$ & $T$ & 1 \\
\hline 220 & 200 & 180 & 160 & 140 & 120 & 100 & 80 & 60 & 40 & 20 & ${ }_{12}^{0} \mathrm{ppm}$ \\
\hline
\end{tabular}




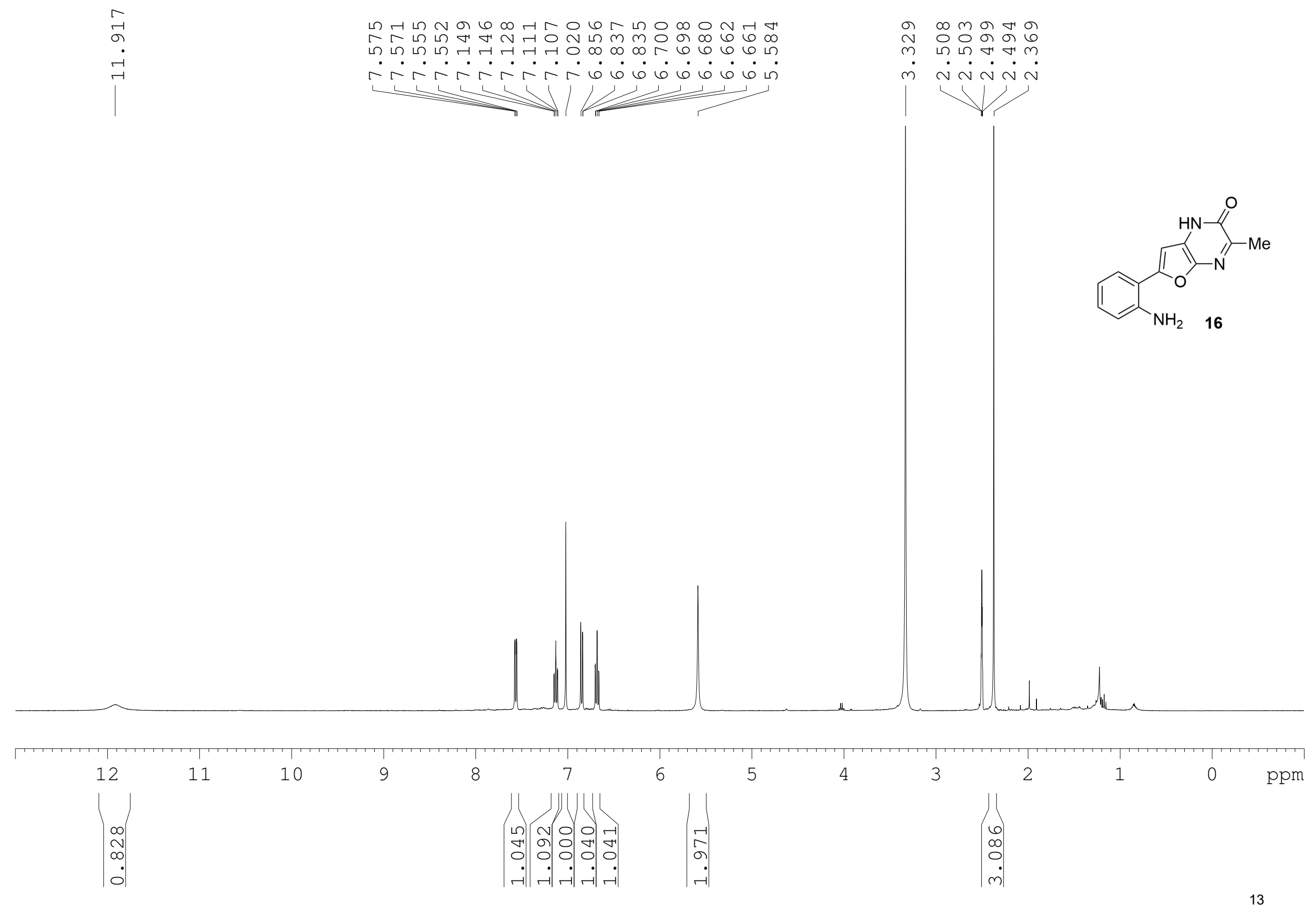




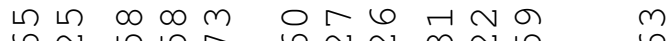

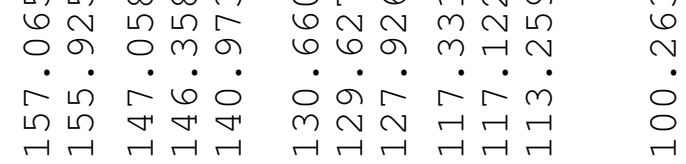

ก

मी

V V

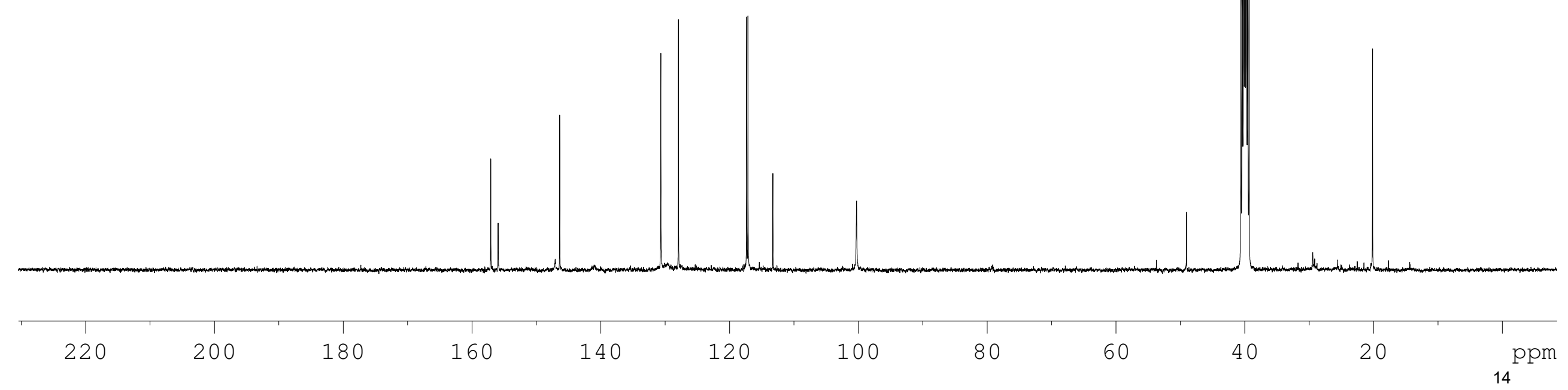




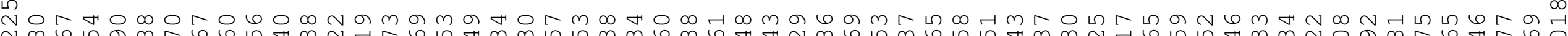
№m

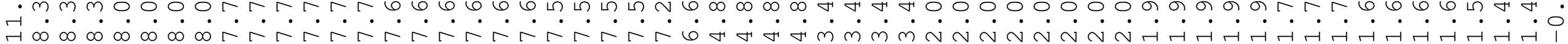

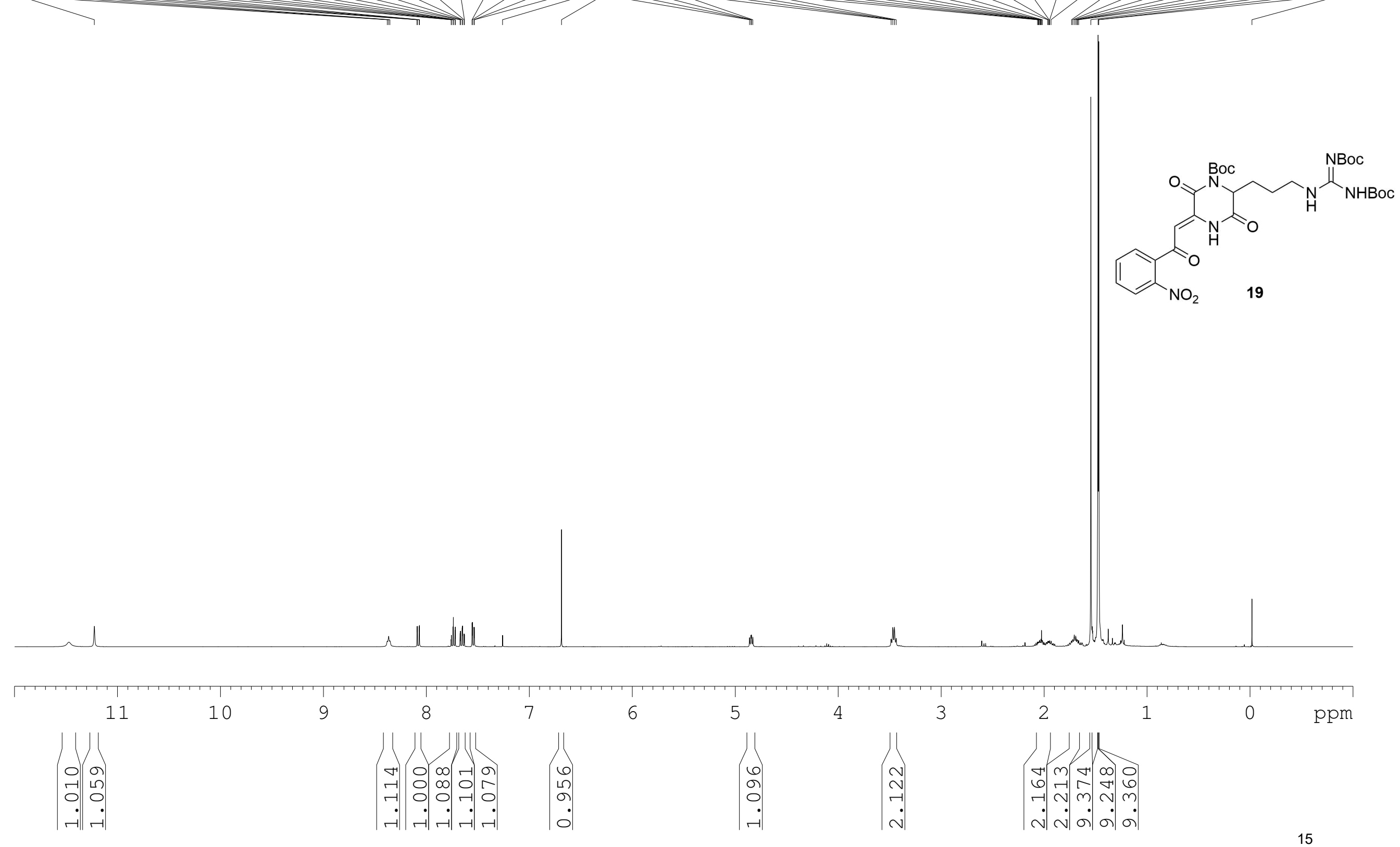




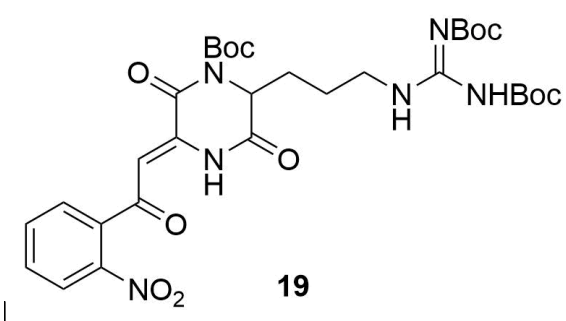

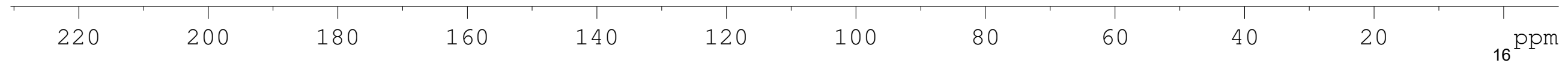




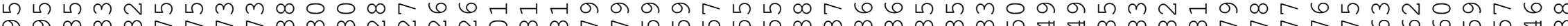

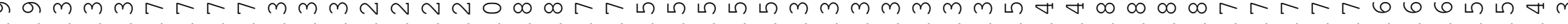

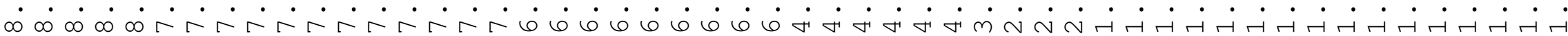

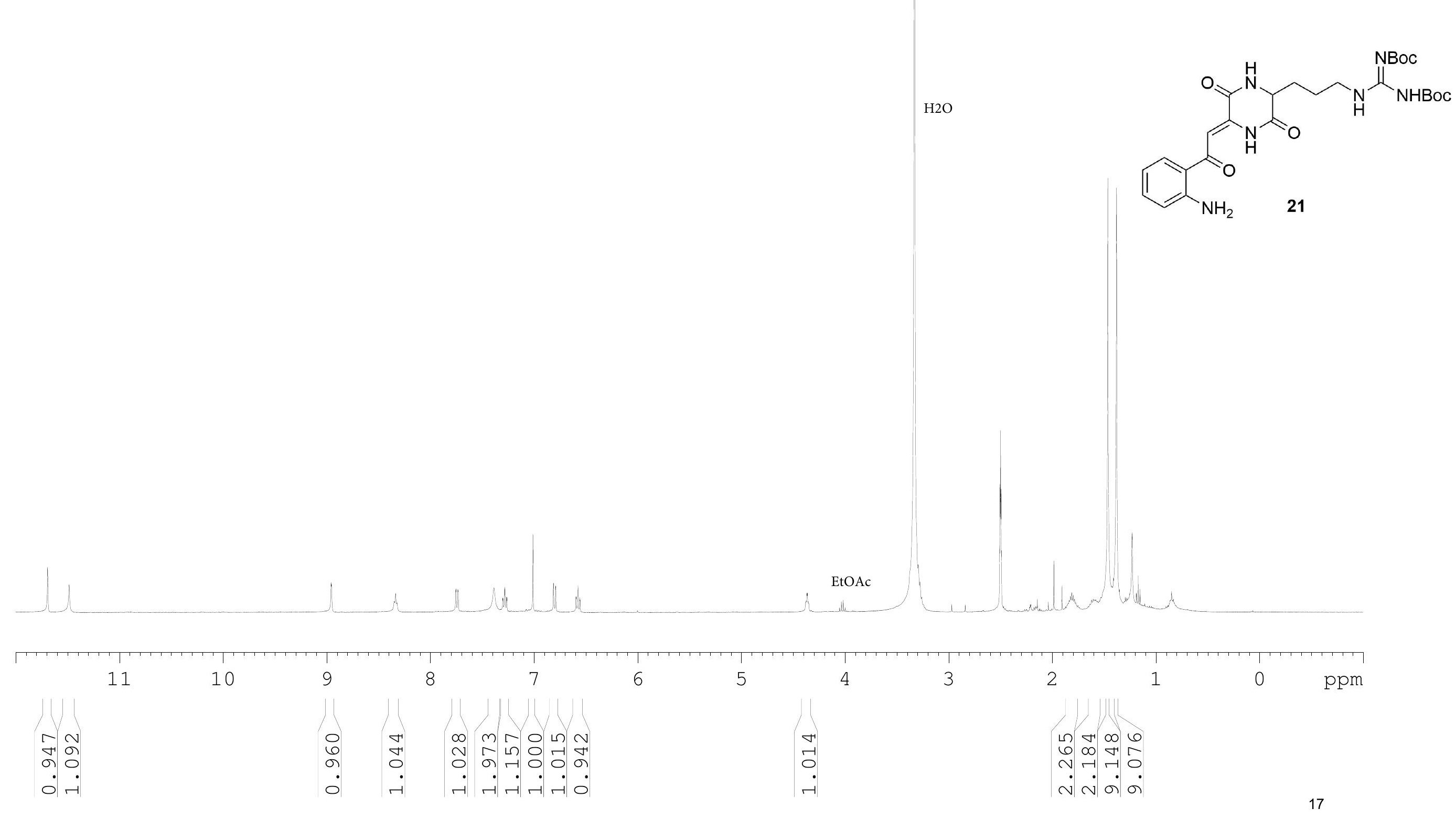




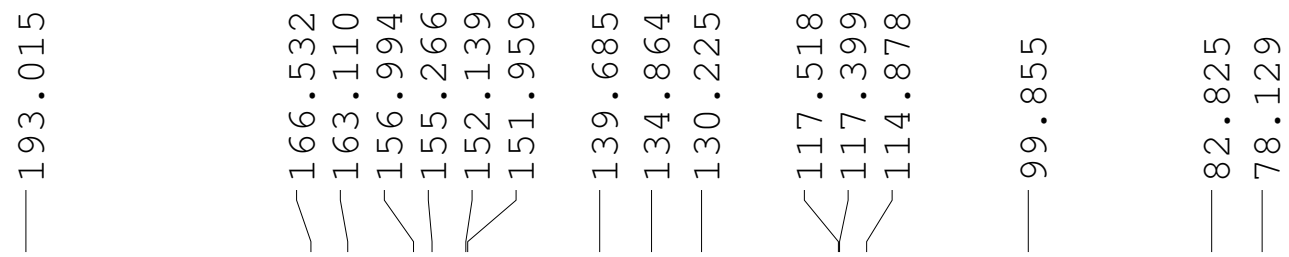

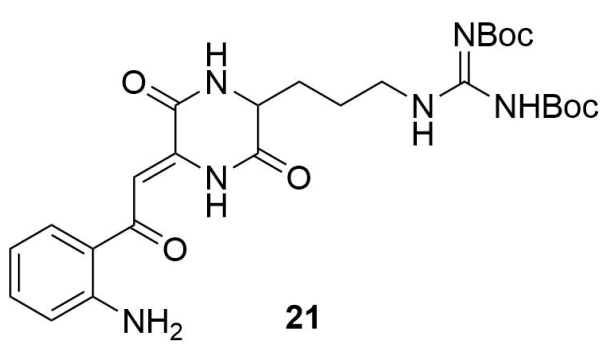

\begin{tabular}{|c|c|c|c|c|c|c|c|c|c|c|c|}
\hline$T$ & $T$ & $T$ & $T$ & $T$ & $T$ & $T$ & $T$ & $T$ & $T$ & $T$ & 1 \\
\hline 220 & 200 & 180 & 160 & 140 & 120 & 100 & 80 & 60 & 40 & 20 & $0_{18} \mathrm{ppm}$ \\
\hline
\end{tabular}




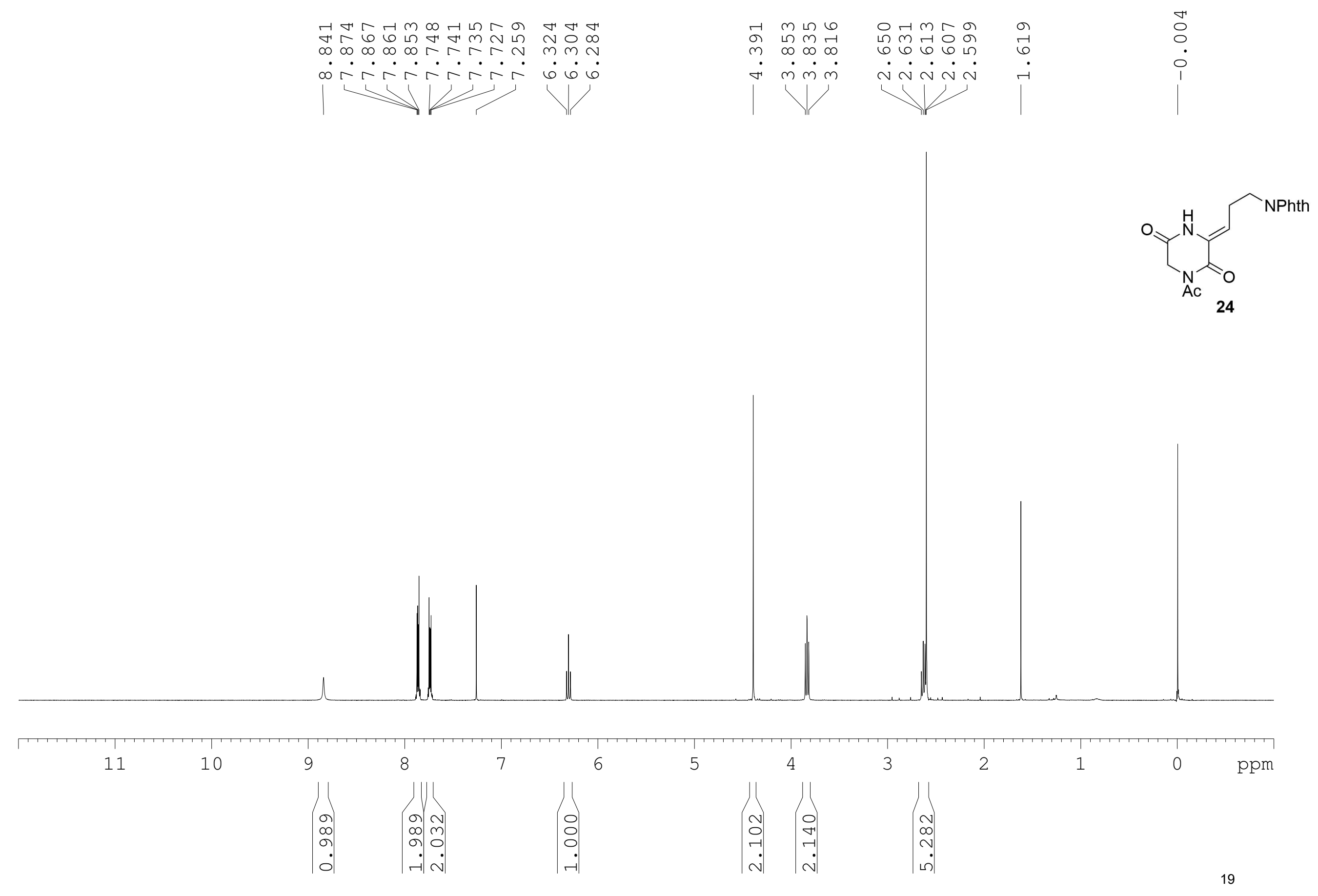




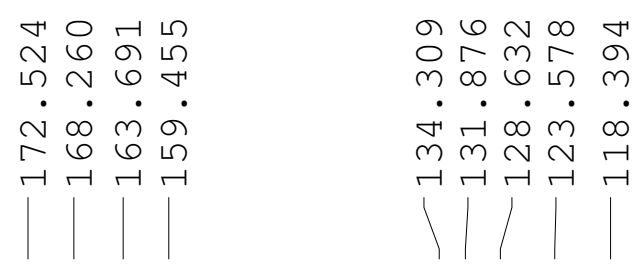

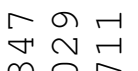
스뭉
I

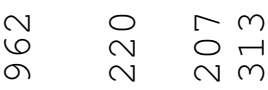

a $\quad \cdots$

๑ $\quad \stackrel{\sim}{\sim} \sim$

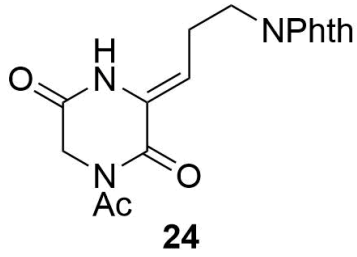

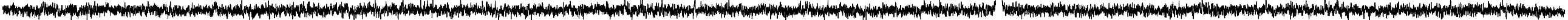

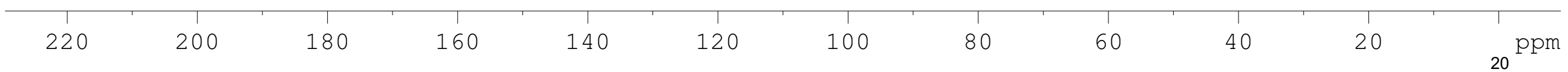




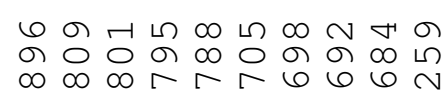

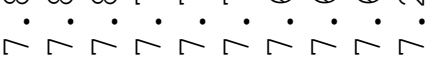

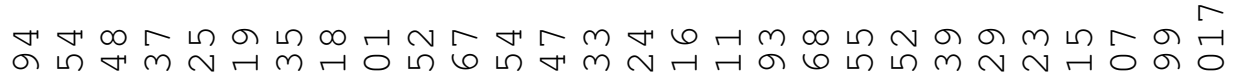
vनन न न न

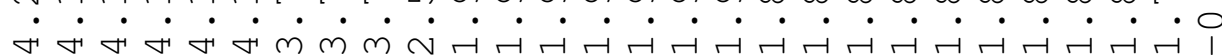

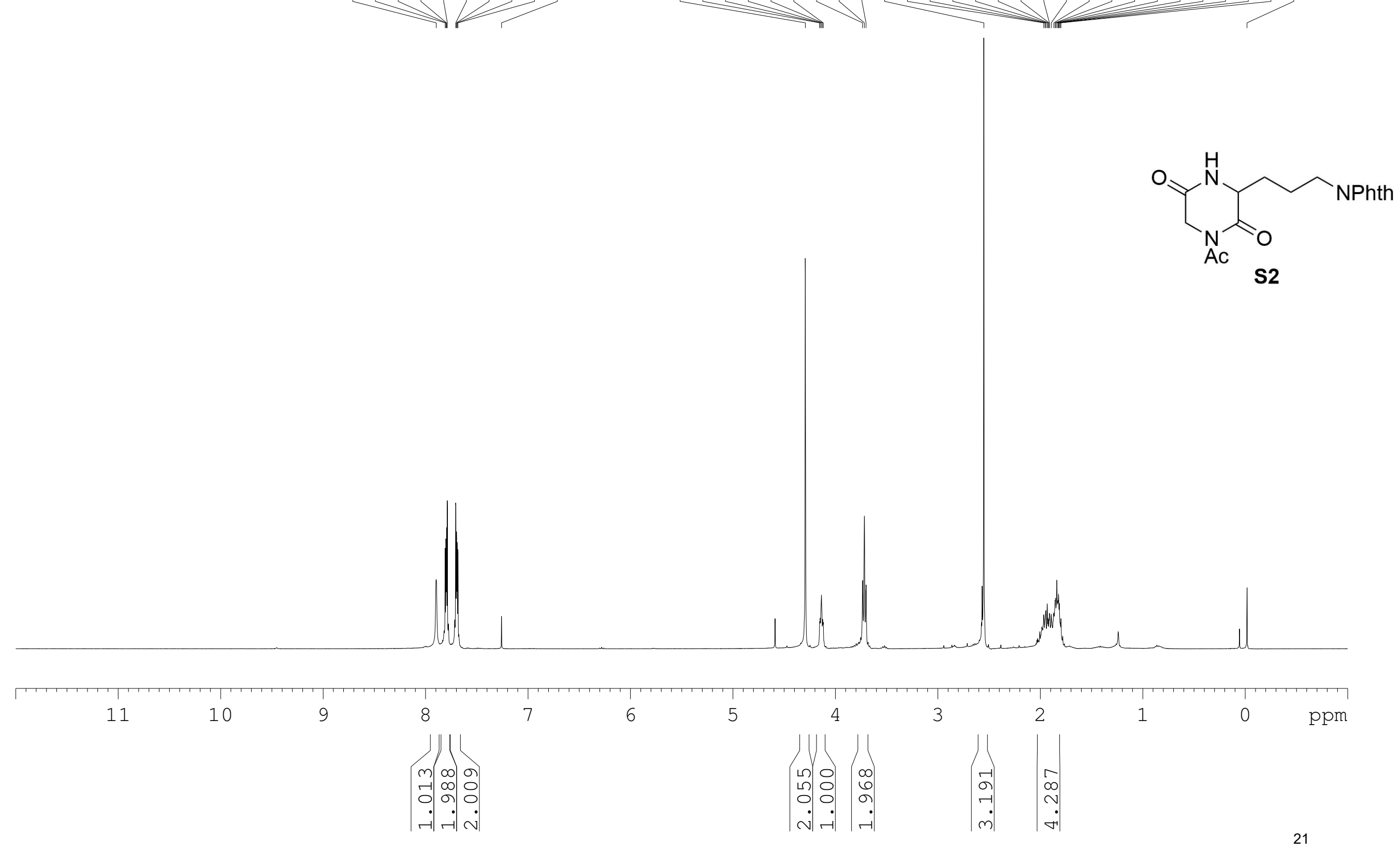




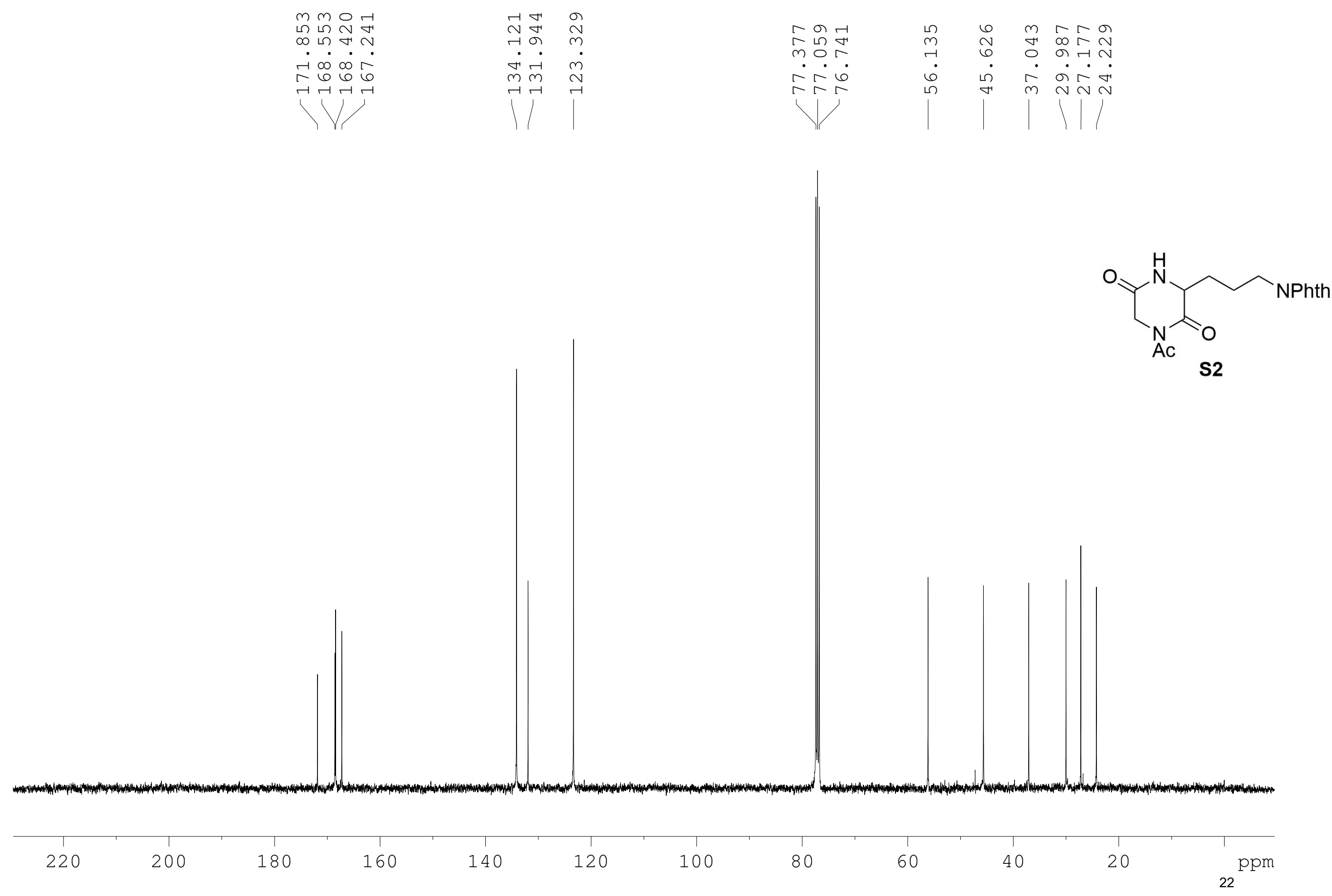




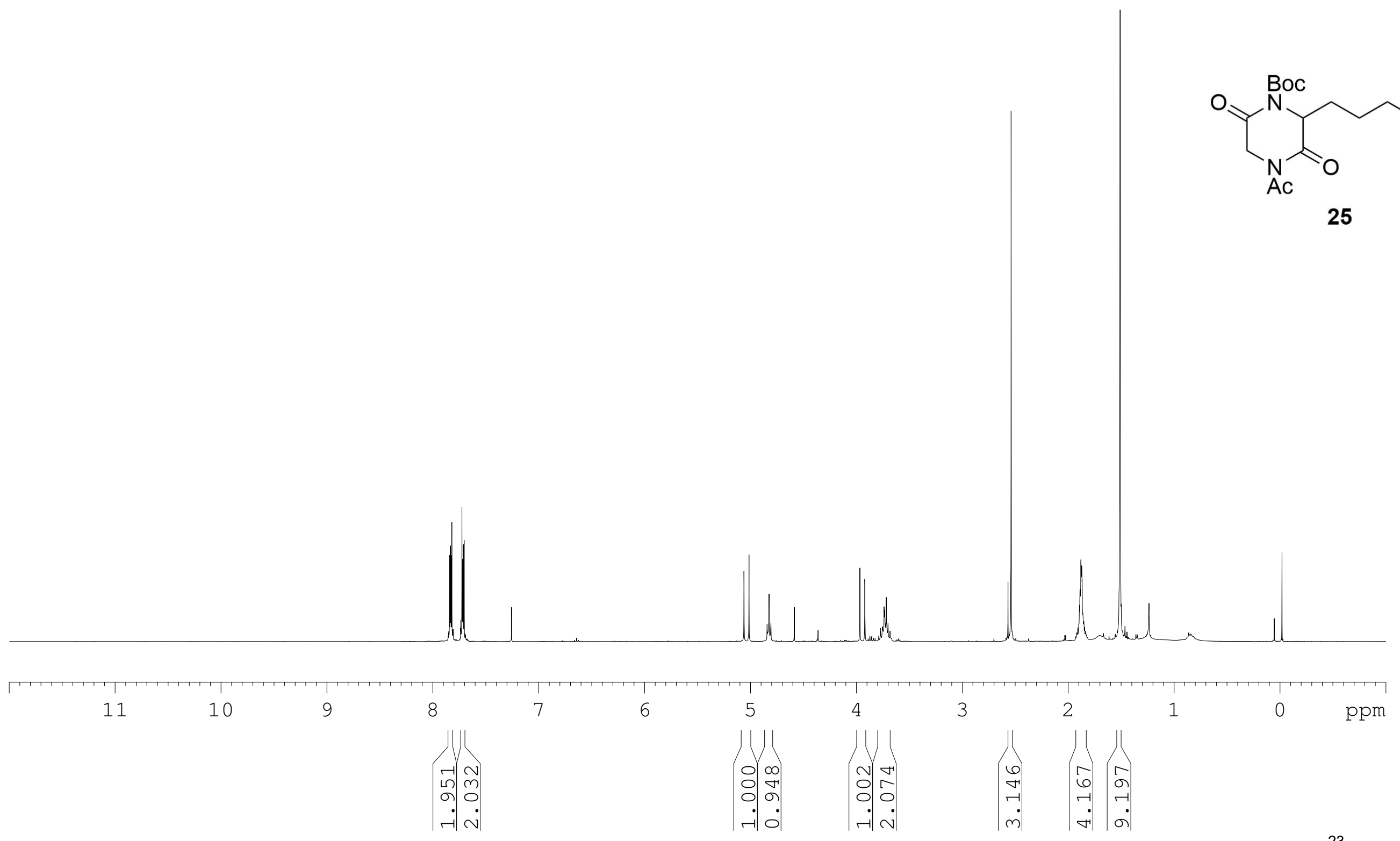

NPhth 


\begin{tabular}{|c|c|c|c|c|c|c|c|}
\hline$N G \Gamma$ & の & の & 6 & & & & \\
\hline$\llcorner\sim \infty \triangleleft$ & $\nabla$ & & $\infty$ & 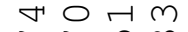 & r & r & $6 \curvearrowleft ナ \sqcup$ \\
\hline$\neg m \omega \infty$ & 0 & 다 & $m$ & 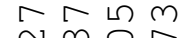 & 덩 & + & $\neg \cong \bigcirc\llcorner N$ \\
\hline$\dot{-i} \dot{\sim} \dot{m}$ & $\dot{0}$ & $\dot{\nabla} \dot{-i}$ & $\dot{m}$ & N mor & 0 & $\sigma$ & ㅁの \\
\hline$\nabla 666$ & 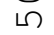 & $m m$ & N & $\curvearrowleft \wedge \sim 6$ & 0 & 6 & r \\
\hline - ન્ન ન્ર & 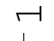 & $\ulcorner\digamma$ & $\Gamma$ & $\infty r r$ & 6 & r & $m m \sim \sim \sim$ \\
\hline
\end{tabular}

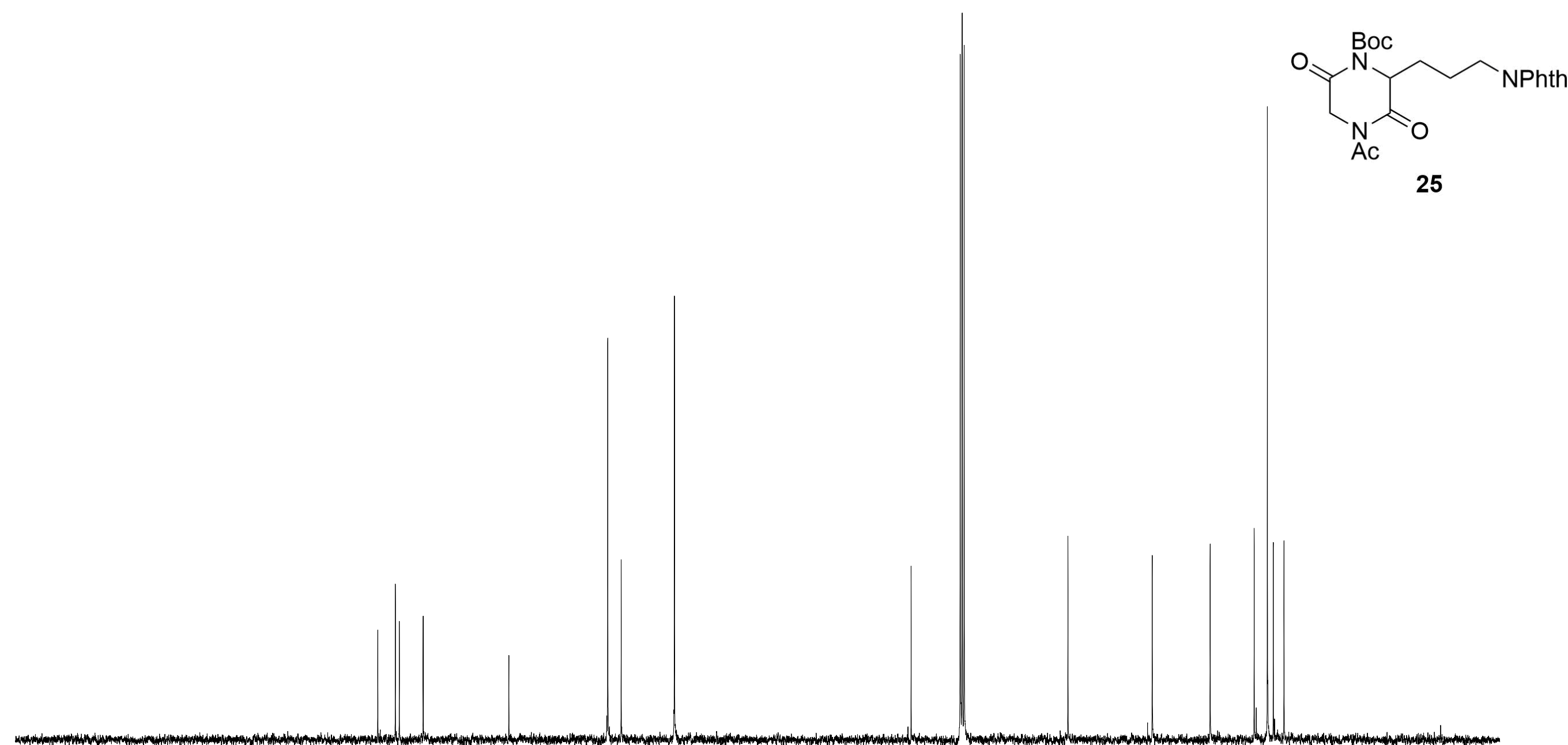

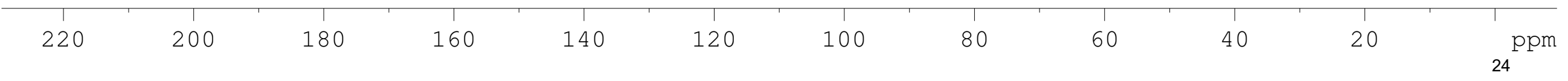


r m $\quad$ 윙ㅁ

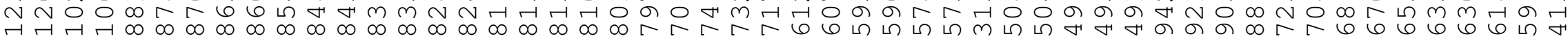

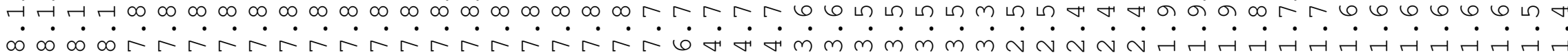

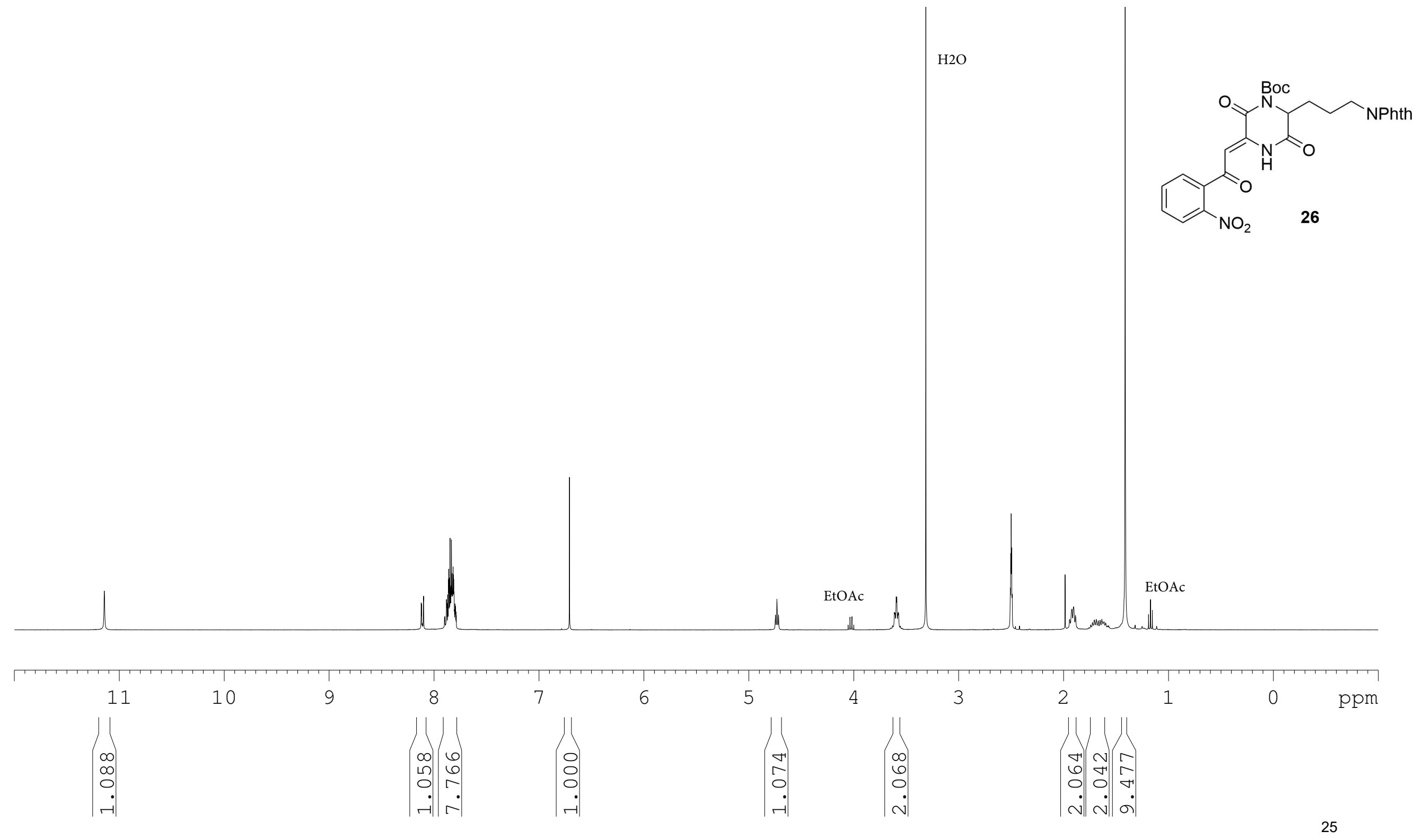




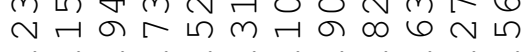

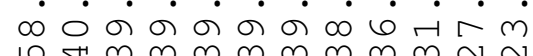

\begin{tabular}{|c|c|c|c|c|c|c|c|c|c|c|c|}
\hline 220 & 200 & 180 & 160 & 140 & 120 & 100 & 80 & 60 & 40 & 20 & $0 \mathrm{ppm}$ \\
\hline
\end{tabular}


EtOAc
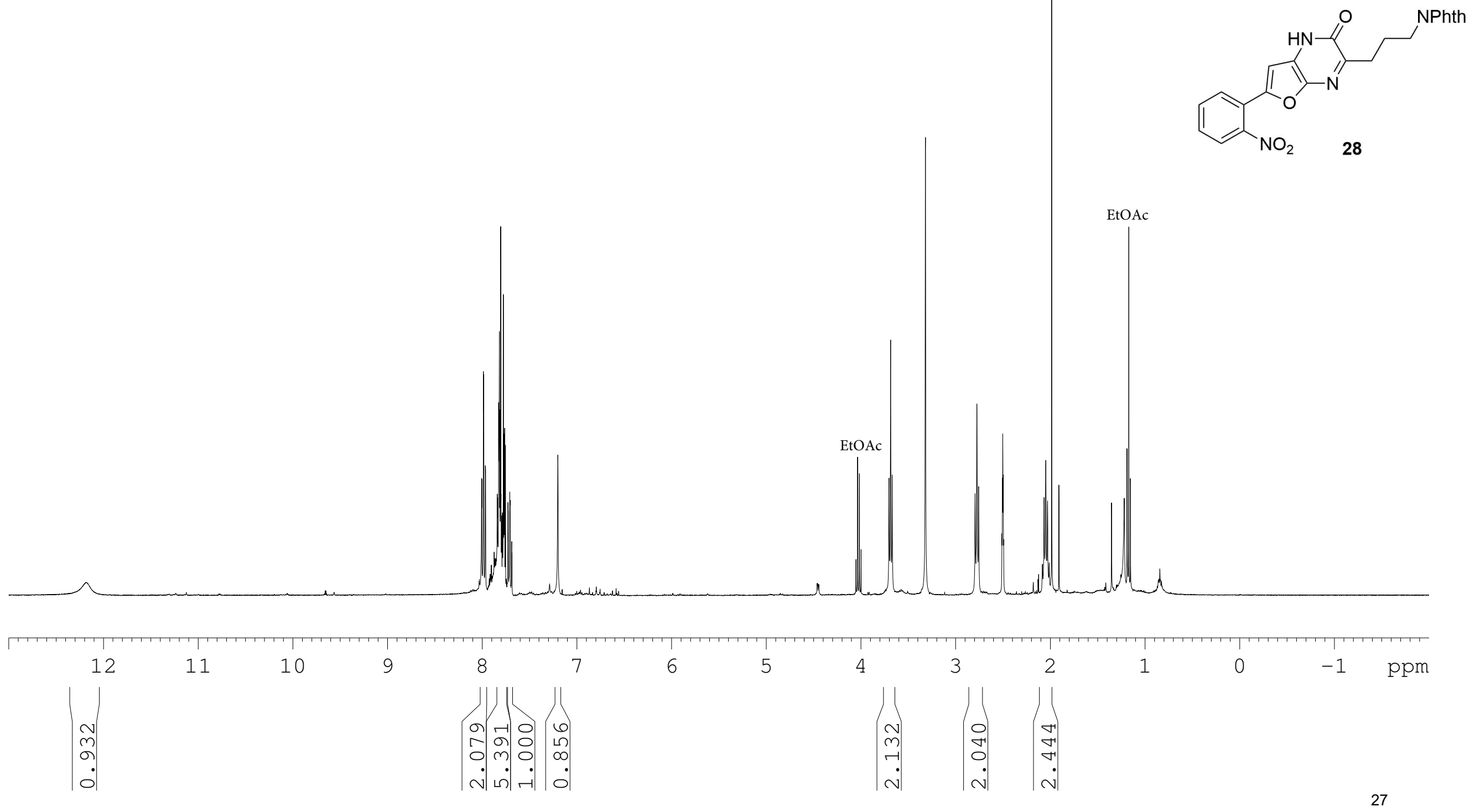


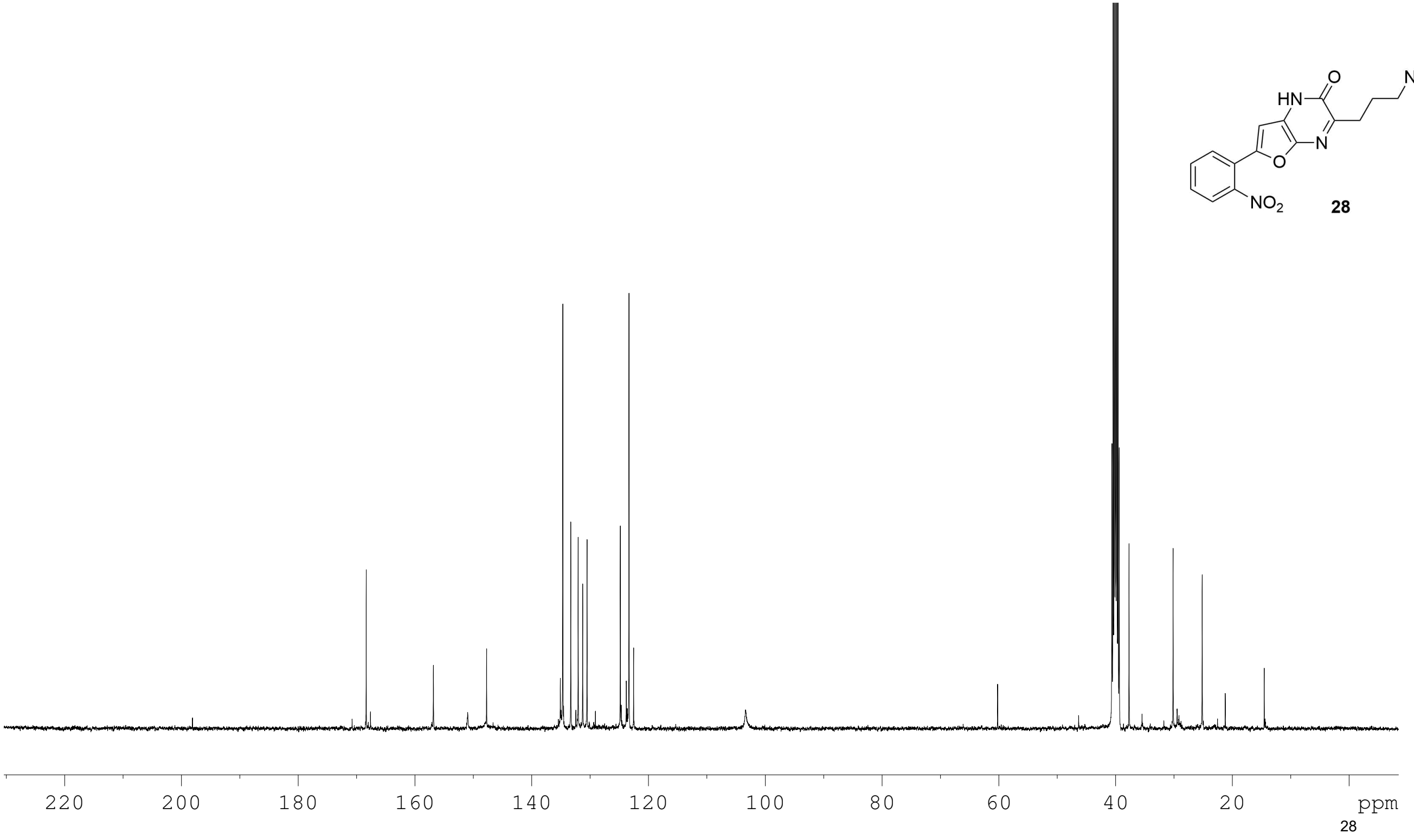




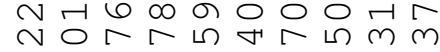

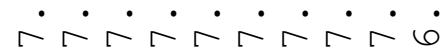

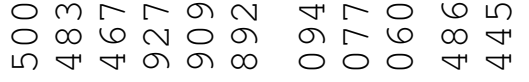

$\dot{m} \dot{m} \dot{\sim} \dot{\sim} \dot{\sim} \dot{\sim} \dot{\sim} \dot{\sim} \dot{r} \dot{r}$

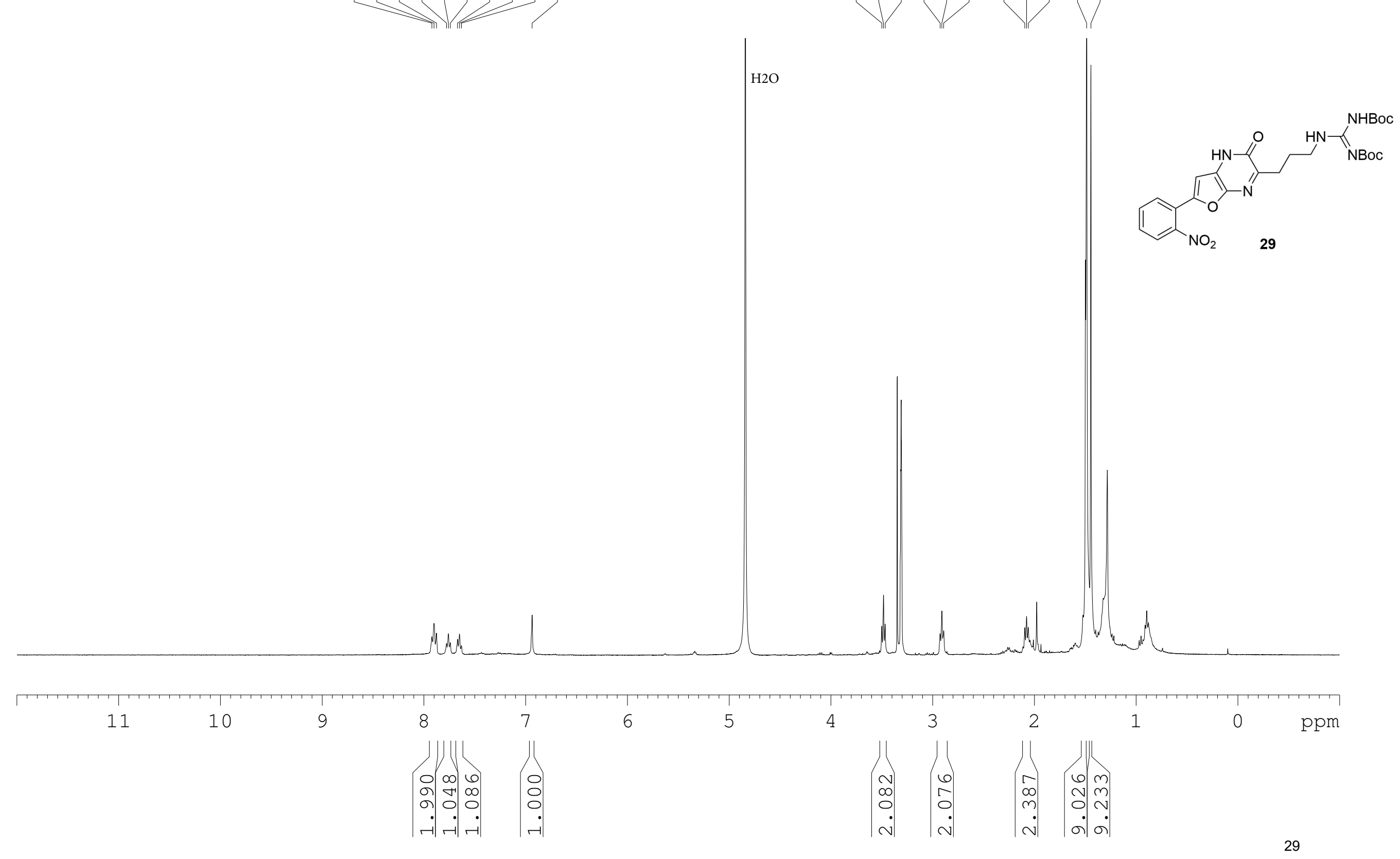


ने न न ब न 60 म m 0 (

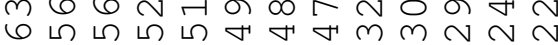

\section{r}

$\circ m \backsim \infty \pi$ ने नंव் $\dot{\circ} \dot{\circ}$ $m \sim \sim \sim \sim$

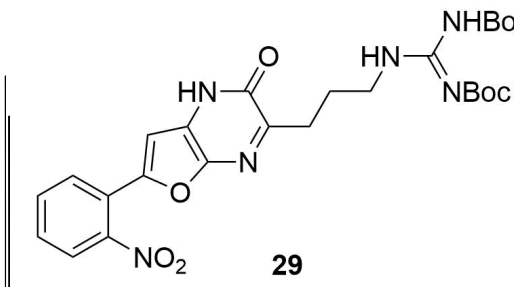

\begin{tabular}{|c|c|c|c|c|c|c|c|c|c|c|c|}
\hline 1 & 1 & 10 & 160 & 1 & 1 & I & 1 & 1 & 1 & 20 & 1 \\
\hline 220 & 200 & 180 & 160 & 140 & 120 & 100 & 80 & 60 & 40 & 20 & ${ }_{30} \mathrm{ppm}$ \\
\hline
\end{tabular}




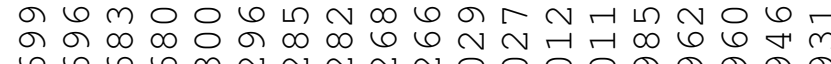

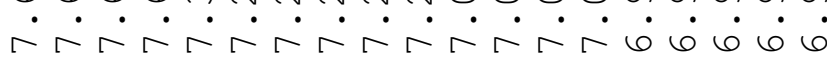

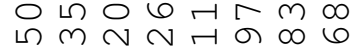
बनवनन००० $\dot{\sim} \dot{\sim} \dot{\sim} \dot{\sim} \dot{\sim} \dot{\sim} \dot{\sim}$

$1 \times \mathrm{CH} 2$ under $\mathrm{MeOH}$

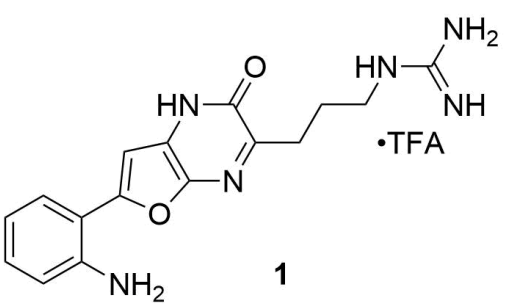

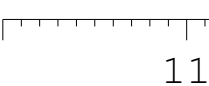

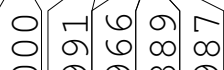

- の

- 


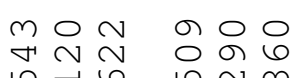

เ.

नें $\dot{\infty} \infty \dot{\infty} \dot{\sigma}$

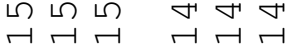

용

a 0 r $m$

$\sim \circ \infty \dot{\circ} \dot{0}$

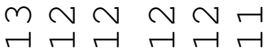

기
m
$\dot{0}$
0
$\dot{1}$

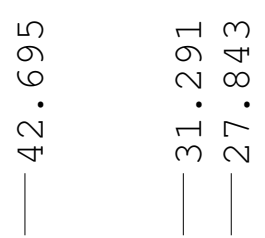
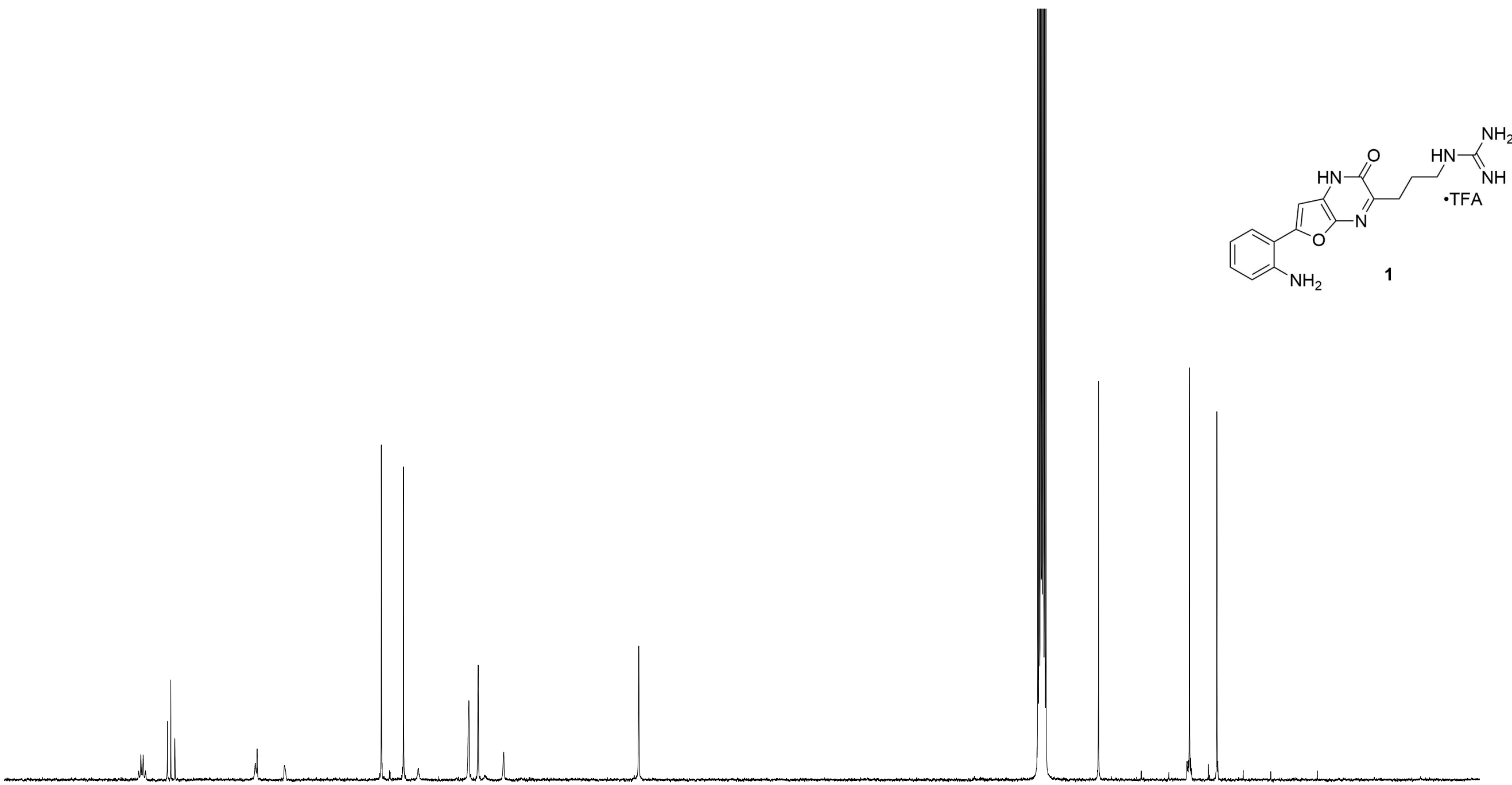


\section{References}

(1) Takahashi, Y.; Iinimura, Y.; Kubota, T.; Tsuda, M.; Sekiguchi, M.; Mikama, Y.; Fromont, J.; Kobayashi, J. Org Lett. 2011, 13, 628-631.

(2) Gaussian 09, Revision D.01, M. J. Frisch, G. W. Trucks, H. B. Schlegel, G. E. Scuseria, M. A. Robb, J. R. Cheeseman, G. Scalmani, V. Barone, B. Mennucci, G. A. Petersson, H. Nakatsuji, M. Caricato, X. Li, H. P. Hratchian, A. F. Izmaylov, J. Bloino, G. Zheng, J. L. Sonnenberg, M. Hada, M. Ehara, K. Toyota, R. Fukuda, J. Hasegawa, M. Ishida, T. Nakajima, Y. Honda, O. Kitao, H. Nakai, T. Vreven, J. A. Montgomery, Jr., J. E. Peralta, F. Ogliaro, M. Bearpark, J. J. Heyd, E. Brothers, K. N. Kudin, V. N. Staroverov, T. Keith, R. Kobayashi, J. Normand, K. Raghavachari, A. Rendell, J. C. Burant, S. S. Iyengar, J. Tomasi, M. Cossi, N. Rega, J. M. Millam, M. Klene, J. E. Knox, J. B. Cross, V. Bakken, C. Adamo, J. Jaramillo, R. Gomperts, R. E. Stratmann, O. Yazyev, A. J. Austin, R. Cammi, C. Pomelli, J. W. Ochterski, R. L. Martin, K. Morokuma, V. G. Zakrzewski, G. A. Voth, P. Salvador, J. J. Dannenberg, S. Dapprich, A. D. Daniels, O. Farkas, J. B. Foresman, J. V. Ortiz, J. Cioslowski, and D. J. Fox, Gaussian, Inc., Wallingford CT, 2013.

(3) Zhao, Y.; Truhlar, D. G. Theor. Chem. Acc. 2008, 120, 215-241. 